\title{
René Geißler
}

\section{Kommunale Unternehmen in der Haushaltskonsolidierung}

Haushaltskrise; Haushaltskonsolidierung; kommunale Unternehmen; Neues Steuerungsmodell; Privatisierung; Rekommunalisierung

Vor dem Hintergrund der kommunalen Haushaltskrise rückten die Unternehmen als historisch gewachsener Bestandteil kommunaler Aufgabenwahrnehmung in den vergangenen zwei Jahrzehnten mehr und mehr in den Blickwinkel der Kommunen. Dieser Beitrag unternimmt einen ersten Schritt, die Zusammenhänge zwischen kommunaler Wirtschaft und Konsolidierung der Kernhaushalte aufzuzeigen. Dies erscheint notwendig, da die Debatte um die Rolle öffentlicher Unternehmen in den Haushaltskrisen der Gebietskörperschaften häufig verkürzt und ideologisch aufgeladen unter dem Schlagwort „Privatisierung “ geführt wird. Dies entspricht jedoch nicht der empirischen Realität, die kreativere und flexiblere Optionen fand, Unternehmen als Pfad der Haushaltskonsolidierung zu interpretieren. Im Ergebnis zeigt sich, dass die finanzstatistisch messbaren Effekte für die Kernhaushalte gering sind. Sehr viel bedeutender sind Entlastungen aus der Reorganisation kommunaler Aufgaben im Konzern. Sie sind jedoch nicht valide zu beziffern. Rekommunalisierung als junger Trend wird primär durch das Auslaufen von Konzessionsverträgen bewirkt. Er folgt jedoch ebenso wirtschaftlichen und haushaltsentlastenden Zielen. Die fiskalische Bedeutung der Unternehmen für die Kommunen ist überdies kein Phänomen der Gegenwart, sondern war stets mit ihnen verbunden.

\section{Einleitung}

Wirft man einen Blick in die kommunalwissenschaftliche Literatur der neunziger Jahre so scheinen drei wesentliche Themen die damalige Diskussion bestimmt zu haben: die gerade begonnene Verwaltungsmodernisierung unter dem Leitbild des Neuen Steuerungsmodells (NSM), die sich ausbreitende Haushaltskrise und die aus der EU-Rechtsetzung resultierende Bedrohung der kommunalen Wirtschaft. ${ }^{1}$ Aus heutiger Sicht stehen diese drei Themen und deren weitere Entwicklung in Zusammenhang. So begünstigte die Haushaltskrise die Verbreitung des NSM als auch das Wachstum der kommunalen Wirtschaft (Reichard 2007). Rückblickend lässt sich feststellen, dass die mit dem NSM verbundenen Hoffnungen in der Kernverwaltung bezüglich Modernisierung und Konsolidierung trotz einiger Erfolge nicht eintraten und die Haushaltskrise ein damals nicht vorstellbares Niveau erreichte (Holtkamp 2008, S. 430). Die angesichts bevorstehender Liberalisierungen geäußerten pessimistischen Szenarien für die kommunale Wirtschaft traten jedoch ebenso nicht ein. Ganz im Gegenteil lässt sich das vergangene Jahrzehnt als ein „Triumphzug“

1 Ein viertes großes Thema mögen die Reformen innerhalb der Kommunalverfassungen gewesen sein, z. B. bezüglich der Monospitze, Konnexität oder direktdemokratischer Elemente. 
kommunaler Unternehmen beschreiben, die mit neuem Selbstbewusstsein, neuen Strategien, gewachsen im personellen Umfang und Wirtschaftlichkeit gestärkt aus diesen Liberalisierungen hervorgingen.

Nichtsdestotrotz haben sich die kommunalen Verhältnisse grundlegend geändert. Es stehen sich nunmehr eine teilweise modernisierte, schrumpfende, finanziell ausgezehrte Kernverwaltung und ein wachsender, lukrativer, managerialistischer Bereich der kommunalen Wirtschaft gegenüber. Das Wachstum der kommunalen Wirtschaft lässt sich verschiedentlich belegen, so zum Beispiel als steigender Anteil der Auslagerungen an der kommunalen Gesamtbeschäftigung, der Investitionen oder auch der Verschuldung, die vormals innerhalb der Kernverwaltung organisiert wurde (Bertelsmann Stiftung 2008). Aus dieser Situation erwachsen Risiken als auch Chancen. Letztere versuchen die Kommunen besonders fiskalisch zu nutzen.

Der Zusammenhang zwischen der Haushaltskrise und dem Wachstum kommunaler Unternehmen, bedingt durch umfangreiche Auslagerungen, wurde häufig statuiert (z. B. Bogumil 2010, S. 60). Ebenso besteht Einigkeit darin, dass Haushaltskonsolidierung für die (größeren) Kommunen ohne Berücksichtigung derer Unternehmen weder möglich noch sinnvoll ist (Schwarting 2006, S. 135 f.). Eine systematische Diskussion dieses für die kommunale Praxis in Vergangenheit und Zukunft wesentlichen Trends unter dem Blickwinkel der Haushaltskonsolidierung hat bisher jedoch nicht stattgefunden.

Dieser Beitrag knüpft an diese Forschungslücke an und unternimmt auf Grundlage vorliegender Publikationen, öffentlicher Statistik und Empirie einen ersten Schritt, die Zusammenhänge zwischen kommunaler Wirtschaft und Haushaltskonsolidierung aufzuzeigen. Der öffentliche und politische Diskurs spiegelt mit seiner Fokussierung auf einen wertenden Privatisierungsbegriff die empirische Realität in den Kommunen nur ungenügend wider. Deren Maßnahmen waren hingegen überwiegend organisatorischer Natur und werden in der Folge als Auslagerung bezeichnet. Im Rahmen dieser Arbeit bedeutet der Begriff Auslagerung allgemein die institutionell-organisatorische und fiskalische Übertragung einer Aufgabe an einen Träger außerhalb der Kernverwaltung, gleich welcher Rechtsform (Killian, Richter, Trapp 2006, S. 20).

Der Beitrag beginnt mit einer kurzen Darstellung der Geschichte kommunaler Wirtschaft (Kapitel 2). Die Historie ist aufschlussreich, um Entwicklungen, Diskussionen und Standpunkte nachzuvollziehen und einzuordnen. In Kapitel 3 wird die aktuelle Lage der kommunalen Kernhaushalte skizziert, die ebenso in engem Zusammenhang zur Entwicklung und Rolle kommunaler Wirtschaft steht. Die Debatte um die Bedeutung kommunaler Unternehmen ist häufig von einer gewissen Empiriearmut begleitet. Kapitel 4 greift daher auf die Zahlen der Gemeindefinanzstatistik des Statistischen Bundesamtes im Zeitverlauf 1997 bis 2007 zurück und diskutiert anhand dieser die monetär messbaren Effekte kommunaler Unternehmen für die Kernhaushalte. Tatsächlich gehen die entlastenden, haushaltskonsolidierenden Erträge weit über diese Statistik hinaus. Kapitel 5 widmet sich daher unter dieser Perspektive den Variationen von Auslagerungen, deren Motivation und Wirkungen für den Kernhaushalt. Schlussendlich verdichtete sich in jüngerer Zeit die Debatte um die Rekommunalisierung vormals ausgelagerter Leistungen. Auch diese steht in engem Bezug zur Haushaltskrise der Kommunen.

Dieser Beitrag erhebt nicht den Anspruch, die Thematik abschließend und allumfassend zu beleuchten. Im Gegenteil soll er als sachliche Grundlage weiterer Diskussion in einem wichtigen Themenfeld dienen, das bis dato weithin als Forschungslücke zu bezeichnen ist. 


\section{Entwicklungstrends der kommunalen Wirtschaft}

Kommunale Unternehmen sind sowohl Wesenselement als auch Resultat kommunaler Selbstverwaltung und historisch untrennbar mit der gemeindlichen Aufgabenerfüllung verbunden. Der Begriff lässt sich empirisch sowohl aus der Eigentümerschaft, bestimmten Aufgaben oder der Legitimation abgrenzen. Allgemein werden hierunter wirtschaftliche Betriebe verstanden, die von Kommunen betrieben bzw. beherrscht werden, um einen öffentlichen Zweck zu erfüllen (Gottschalk 1994, S. 217). In der wirtschaftlichen Tätigkeist sind den Kommunen über die Gemeindeordnungen Grenzen gesetzt (vgl. Schwarting 2001).

Kommunale Unternehmen unterliegen einer großen Aufgaben- und Organisationsvielfalt. Sie sind nicht immer an äußeren Merkmalen erkennbar (Edeling 2004, S. 9). In jüngerer Zeit erfuhren die kommunalen Unternehmen einen Bedeutungszuwachs und rückten verschiedentlich in das öffentliche Interesse. In den vergangenen zwanzig Jahren war im Zuge umfangreicher Auslagerungen eine starke Zunahme der Formenvielfalt zu beobachten. Ein wachsender Anteil an Beschäftigung und Investition wird in Unternehmen erbracht (vgl. Bertelsmann Stiftung 2008).

Die Geschichte der kommunalen Wirtschaft verlief von Anbeginn nicht einheitlich, sondern war von mehreren Paradigmenwechseln begleitet. Sie entstand in der zweiten Hälfte des 19. Jahrhunderts im Zuge der Industrialisierung und Urbanisierung aus der Erkenntnis, dass die Privatwirtschaft in zentralen Aufgaben der Daseinsvorsorge nicht zu einer befriedigenden Versorgung der Bevölkerung führte (vgl. Ambrosius 2003). ${ }^{2}$ Die Motive der damaligen Kommunalisierung von Aufgaben und Betrieben waren vielfältig. Übergeordnet stand der schnelle, flächendeckende und soziale Aspekte berücksichtigende Ausbau der Infrastruktur. Wirtschaftliche Basisfaktoren, die den Charakter natürlicher Monopole annahmen, sollten öffentlich organisiert werden, um Missbrauch zu vermeiden und Entwicklung zu befördern (Röber 2009, S. 75 ff.). Bereits in dieser Zeit waren jedoch auch fiskalische Aspekte von erheblicher Bedeutung (Bogumil et al. 2010, S. 9 f.). So erhofften sich die Kommunen besonders im Bereich der Ver- und Entsorgung eine von Gewinnen begleitete Aufgabenerfüllung.

Kommunale Unternehmen dienen dem Zweck und erlangen ihre Legitimation daraus, kommunale Aufgaben wirtschaftlich und entsprechend der durch den Stadtrat gesetzten Ziele umzusetzen. Kommunale Unternehmen sind primär Instrumente zur Verwirklichung politisch bestimmter Aufgaben in Branchen, in denen das Marktprinzip nicht oder nicht ausreichend funktioniert. Der Zielkatalog ist fast unendlich, mehrdimensional und darf sich nicht in der fiskalischen Funktion der Einnahmegenerierung erschöpfen. Auch aus diesem Grund beschränken die Gemeindeordnungen das Branchenspektrum kommunaler Unternehmen (Schwarting 2001, S. 294 f.).

Die neunziger Jahre standen unter dem Zeichen der Auslagerung kommunaler Aufgaben. Rückblickend können vier Trends als Treiber dieser Auslagerungen betrachtet werden, welche sich teilweise gegenseitig verstärkten (Abbildung 1). Zum einen führte das NSM, begünstigt durch Haushaltskrise und Unzufriedenheit mit der traditionellen bürokratischen Organisation, zu einer verstärkten Auslagerung kommunaler Aufgaben mit der Erwartung, diese außerhalb der Kernverwaltung wirtschaftlicher und kundenfreundlicher erfüllen zu können. Das NSM in Gänze war

2 Zu den Entwicklungsstufen der Kommunalwirtschaft vgl. Rollenfisch 2007. 
daher auch durch eine gewisse Skepsis gegenüber öffentlicher Verwaltung als auch einem diesbezüglichen Leitbildwandel motiviert (vgl. Jann 2010). Obgleich unmittelbar Bestandteil des NSM, können Auslagerungen auch als Eingeständnis des Scheiterns der Binnenmodernisierung der Kernverwaltung interpretiert werden. Von einem Wechsel der Rechtsform erhoffte man sich eine quasi automatische Angleichung der Handlungsrationalitäten von einer bürokratischen zu einer wirtschaftlichen. Über die höhere Wirtschaftlichkeit sollte der Haushalt entlastet werden. Die Haushaltskrise wird daher gemeinhin als der stärkste Einfluss betrachtet (Bogumil 2010, S. 60). Mit dem NSM dezentralisierte sich die Kommunalverwaltung und es entstand eine ,institutionelle Vielfalt" kommunaler Aufgaben. Demgegenüber ließe sich auch ein indirekter Zusammenhang zwischen dem Leitbild NSM und der Deregulierungspolitik der EU feststellen. Jene lässt sich aus dem seit Anfang der achtziger Jahre im angelsächsischen Raum präsenten New Public Management begründen, welches einige Jahre später in der deutschen Adaption NSM mündete.

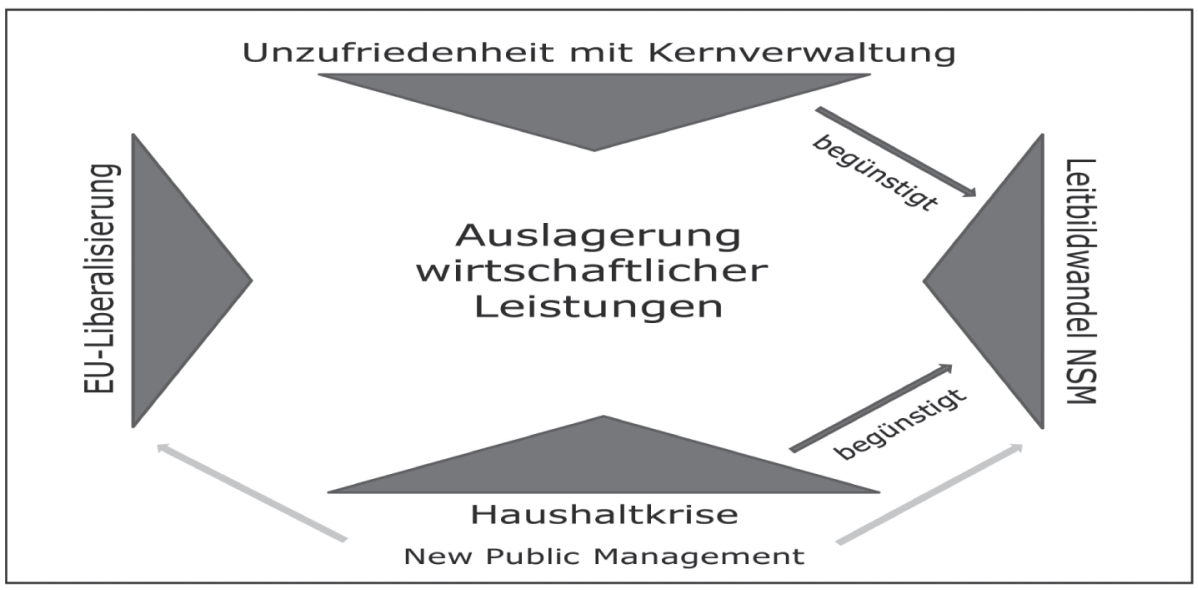

Abbildung 1: Einflussfaktoren auf die Auslagerungen wirtschaftlicher Leistungen in Kommunen Quelle: Eigene Darstellung

Mitte der neunziger Jahre begann mit der Binnenmarktrichtlinie Strom und Gas der EU die Liberalisierung zentraler Branchen der kommunalen Wirtschaft. Das hiermit verfolgte Ziel der Stärkung des Wettbewerbs über die Öffnung ehemals kommunaler Märkte durch den Abbau marktbeschränkender Regulierung wurde als Herausforderung und Gefahr für die deutsche kommunale Wirtschaft gesehen (Reichard 2000, S. 23; Schwarting 2001, S. 293; Bogumil et al. 2010, S. 12).

Nunmehr sind kommunale Monopole als wettbewerbliche Ausnahmebereiche nur noch in abschließend definierten Branchen zulässig (vgl. Schwintowski 2003). An die Frage der wettbewerblichen Ausnahmebereiche schloss sich die Diskussion um kommunale Querverbünde an. Selbige waren üblich, um Mittels der Gewinne ertragsstarker Unternehmen die Verluste defizitärer Leistungen (z. B. ÖPNV) auszugleichen. Der kommunale Querverbund wurde von Seiten der Privatwirtschaft lange Zeit bekämpft (Oettle 1994, S. 297 ff.). Er wurde als verdeckte Sub- 
vention durch monopolisierte Betriebe gesehen. Die EU folgte der Kritik in Teilen und begrenzte die für den Querverbund zulässigen Branchen. Überhaupt wird seit jeher eine auch ideologisch basierte Diskussion um die etwaige Privilegierung kommunaler Unternehmen geführt. Selbige sehen Kritiker neben dem Querverbund auch in Gebietsmonopolen oder der Haftung der Kommune (vgl. Püttner 1994). Unabhängig der Bewertung dieser Diskussion wurden in den letzten Jahren einige dieser Privilegien durch die EU abgebaut. Spiegelbildlich wurden der Querverbund und die damit gegebenen Finanzierungsquellen nicht kostendeckender Leistungen geschwächt. Die Liberalisierungen der EU lösten in der kommunalen Wirtschaft Prozesse der Konzentration, Kooperation und neuer Geschäftsstrategien aus (Leonhardt 2000, S. 61; Gottschalk 2003, S. 202). Letztlich funktioniert der Wettbewerb bezüglich der privaten Endverbraucher nur bedingt (Monopolkommission 2009, S. 73 ff., S. 88). Die lokalen Märkte und stabilen Erträge blieben weitgehend erhalten.

Seit jeher werden kommunale Unternehmen von privater Seite als systemfremdes Element der Marktwirtschaft kritisiert und auch normativ infrage gestellt (Löwe 2001, S. 414; Ambrosius 1994, S. 203; Henke et al. 2007, S. 31). Argumente für die Ineffizienz öffentlicher Unternehmen wurden sowohl aus der ökonomischen Theorie der Politik, der Bürokratietheorie oder der Institutionenökonomie hergeleitet (Budäus 2000, S. 21 f.). Der empirische Nachweis ist jedoch offen (vgl. Mühlenkamp 2001).

Aus der Organisationshoheit der Kommunen und dem Prinzip der Örtlichkeit resultieren ein kaum überschaubares Spektrum kommunaler Unternehmen hinsichtlich Branchen oder Aufgaben ebenso wie eine nicht abschließend definierbare Anzahl. ${ }^{3}$ Darüber hinaus entstehen kommunale Unternehmen beständig neu. Aktuellen Erhebungen zufolge haben im Jahr 2009 rund 90\% der kommunalen Unternehmen eine private Rechtsform (vgl. Papenfuß 2010). Die überwiegende Mehrheit ist den historisch gewachsenen Branchen der Ver- und Entsorgung zuzuordnen.

Die mit den Auslagerungen einhergehenden Prozesse der Konzernbildung und deren Konsequenzen wurden lange Zeit vor dem Hintergrund des NSM wenig beachtet (Bremeier 2000, S. 326). Es entstanden latent intransparente Konzernstrukturen, in denen die Kernverwaltung quantitativ beständig an Gewicht verlor. Aus der sich häufenden Einsicht in die Steuerungsmängel der Auslagerungen entwickelte sich, meist im Geschäftsbereich der Kämmerei, das Beteiligungsmanagement (Bogumil et al. 2007, S. 75).

Die durch NSM, Haushaltskrise, Unzufriedenheit mit der tradierten Verwaltung und Liberalisierung ausgelöste Ökonomisierung der Kommunalverwaltung und die zunehmende Ausrichtung an privatwirtschaftlichem Handeln und Rationalitäten wird vermehrt kritisch gesehen (vgl. Harms, Reichard 2003). Die Ursachen dieser Ökonomisierung liegen in den sich wandelnden ökonomischen und rechtlichen Rahmenbedingungen, die nicht ohne Auswirkungen auf Kommunen und ihre Unternehmen bleiben konnten. So hat die Liberalisierung wohl zu höherer Wirtschaftlichkeit geführt, die Markt- und Kundenorientierung gestärkt, unweigerlich aber die Entfremdung der Unternehmen vom öffentlichen Auftrag und Eigentümer forciert und somit die politische Steuerbarkeit erschwert (vgl. Edeling 2004). Die Evaluation des NSM verdeutlicht, dass Ausgliede-

3 Das Statistische Bundesamt zählt für das Jahr 200813.208 kommunale Unternehmen, Fonds und Einrichtungen mit kaufmännischem Rechnungswesen. Vgl. Datenreport 2011, S. 92. Henke et alteri 2007, S. 30 schätzen den Beschäftigungsumfang kommunaler Unternehmen auf ca. 530.000 Beschäftigte. Zahlen sind jedoch stets abhängig von den betrachteten Branchen und Rechtsformen. 
rungen zu erheblichen Steuerungsverlusten und zu einer Fragmentierung kommunaler Aufgabenerledigung geführt haben (Bogumil et al. 2007, S. 77). Aus Sicht der kommunalen Wirtschaft besteht ein Widerspruch zwischen Behauptung im Markt und politischen Steuerungsansprüchen (Edeling 2003, S. 247). Diese Steuerungsprobleme sind umso größer, je marktfähiger und damit lukrativer die entsprechende Leistung ist. Die Machtverschiebung aus der Kernverwaltung heraus schwächt die politische Funktion der Stadträte, schafft neue Abhängigkeiten der Kommunen von wenigen lukrativen Unternehmen und mindert parlamentarische Kontrolle (Bogumil, Holtkamp 2002). Die Instrumentalfunktion der Umsetzung öffentlich bestimmter Aufgaben, grundlegende Legitimation kommunaler Unternehmen, wird über die Ökonomisierung und Autonomie erschwert. Öffentliches Eigentum an einem Unternehmen bedeutet nicht mehr automatisch die Erfüllung derer öffentlicher Aufgaben. Diesen Gefahren kann durch eine dementsprechend ausgestaltete Steuerung entgegengewirkt werden, welche sich ebenso beständig an sich wandelnde Rahmenbedingungen anpassen muss und tendenziell anspruchsvoller wird (Bremeier 1998, S. 291). So sind Beteiligungsmanagement und in jüngerer Zeit Public Corporate Governance zwei Reaktionen auf die durch NSM und Liberalisierung forcierte Konzernbildung (vgl. Hammerschmid 2009). Deren empirische Verbreitung und Anwendung wird ihrer Bedeutung jedoch noch nicht gerecht.

Diese Entwicklungstendenzen haben tiefe Spuren in den Kommunalverwaltungen hinterlassen, die weit über die Frage der Rechtsformen hinausgehen.

\section{Die Haushaltskrise der Kommunen}

Haushaltsdefizite begleiten die Kommunen der Bundesrepublik bereits seit Jahrzehnten. Traten sie in den siebziger und achtziger Jahren eher vereinzelt und in strukturschwachen Regionen auf, so wurden sie in den neunziger Jahren zu einer flächendeckenden Konstante mit beständig steigendem Problemdruck. Ein gängiger Indikator der Haushaltskrise sind die Kassenkredite (vgl. Abbildung 2). Sie dienen theoretisch der bloßen Liquiditätssicherung und sind von den originären Investitionskrediten zu unterscheiden. Der Anstieg der Kassenkredite belegt, dass es vielen Kommunen trotz intensiver Konsolidierungsmaßnahmen und strenger Rechtspflicht nicht mehr möglich ist, ihre (Kern)haushalte auszugleichen. Bis Anfang der neunziger Jahre quasi in wenig relevanten Größenordnungen, vervierfachten sie sich in den vergangenen zehn Jahren auf ein Niveau, das vormals schlicht nicht vorstellbar erschien. Umso problematischer ist die Feststellung, dass die Kassenkredite sich von der wirtschaftlichen Entwicklung entkoppelt haben und auch in konjunkturell guten Phasen weiter zunehmen. 


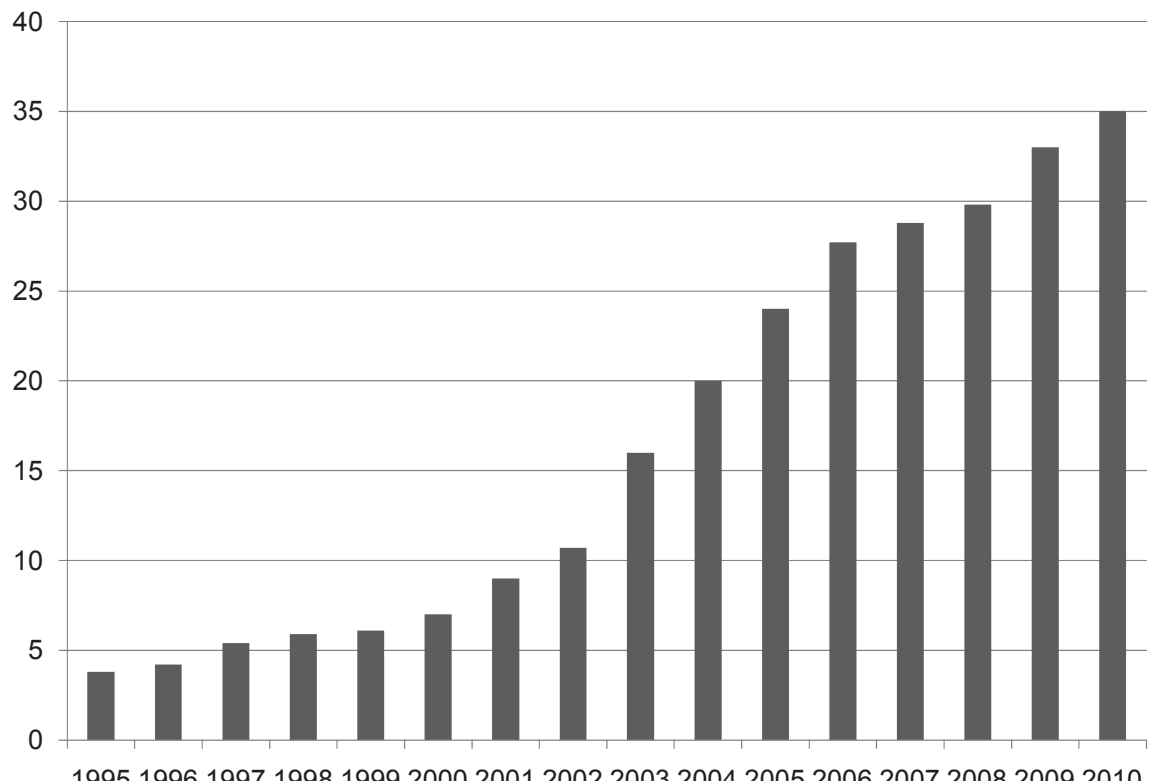

Abbildung 2: Entwicklung der kommunalen Kassenkredite 1995 bis 2010, in Milliarden Euro

Quelle: Deutscher Städtetag

Hinter dem Anstieg der Kassenkredite verbirgt sich als weiterer Problemindikator ein Rückgang der Investitionskredite, die Verschuldungsstruktur verschlechtert sich (vgl. Bertelsmann Stiftung 2008). Die Konsequenzen sind gravierend. Sie drücken sich unter Anderem in einem Verfall der Infrastruktur aus. Die sozialen und politischen Folgen gehen weit darüber hinaus. Je nach wirtschaftlicher Situation der Städte und finanziellen Handlungsspielräume entwickeln sich Lebensverhältnisse und Chancen der Bevölkerung unterschiedlich. Ein Verlust materieller Angebote verstärkt die Trends räumlicher Segregation der Bevölkerung. Es ergibt sich das sozialpolitische Paradoxon der Konzentration von Bevölkerungsgruppen mit besonders hohem Bedarf an öffentlichen und kommunalen Angeboten in Städten, denen hierzu die Handlungsspielräume fehlen. Mit der Veränderung der Bevölkerungsstruktur geht nicht allein ein weiterer Verlust kommunaler Einnahmen, sondern darüber hinaus auch ein Verlust an Humankapital, an aktiver Bürgerschaft und wiederum Gestaltungspotenzialen einher. Schlussendlich häufen sich die sozialen Problemlagen, verschärfen sich die Defizite, sinken kommunale Handlungsspielräume und geraten die Kommunen in eine Vergeblichkeitsfalle, aus der eine endogene Trendumkehr nicht mehr möglich ist.

Zu den sozialen treten die politischen Folgen der Verschuldung. Mit der Zunahme der Haushaltskrise ging eine deutliche Verschärfung des Haushaltsrechts in den Bundesländern einher, welche die Handlungsspielräume defizitärer Kommunen einschränkt und den Einfluss der Rechtsaufsicht spiegelbildlich erweitert (Geißler 2009, S. 70 ff.). Defizitäre Kommunen verlieren durch die Rechtspflicht, den Haushalt kurzfristig über Einnahmeerhöhungen oder Ausgabenkürzungen 
wieder auszugleichen, einen Großteil ihrer Gestaltungsfähigkeit. Diese Konsolidierungsmaßnahmen drücken sich typischerweise aus in Personalabbau, Vermögensveräußerung, Investitionsrückgang, der Schließung von Einrichtungen, Kürzung von Zuweisungen und der Erhöhung von Gebühren oder lokaler Steuern. Besonders die freiwilligen Leistungen betreffen häufig Politikfelder wie Bildung, Kinderbetreuung, Sport, Kultur oder Soziales. Sie sind nicht nur wichtige Kriterien kommunaler Lebensqualität und Profilierungsgebiet der Lokalpolitik, sondern stellen Investitionen in die Zukunftsfähigkeit der Gemeinde dar. Diese Maßnahmen reduzieren wirkliche politische Gestaltung im Stadtrat und führen im Gegenteil zu erhöhten Konflikten. Das Hinterfragen von Besitzständen, Strukturen, Haushaltsstellen und Aufgaben unterbleibt zugunsten pauschaler Kürzungen oder scheinbar unpolitischer Lösungen. Mit dem NSM rückte im Verlauf der neunziger Jahre die Verwaltungsmodernisierung in den Fokus, deren wesentliche Motivation, die Hoffnung auf Kürzungsbeiträge, sich jedoch nur bedingt erfüllte (Bogumil et al. 2007, S. 83 ff.). Gleichwohl die kommunale Finanzkrise allenthalben beklagt wird, ist ihr tatsächlicher Umfang kaum messbar. Denn eine Folge des Trends der Auslagerungen ist die extreme Zersplitterung der kommunalen Haushalte in einen Kernhaushalt und je nach Größe der Stadt Dutzende separate Haushalte der Beteiligungen. Während Erstere seit Jahren defizitär sind, wurden in Letzteren die wirtschaftlichen Leistungen gebündelt. Da bis dato ein konsolidierter Gesamtabschluss der Kommunen (meist) fehlt, ist nicht transparent ersichtlich, wie sich der kommunale Gesamthaushalt im Einzelfall darstellt (vgl. Budäus, Hilgers 2009; Bertelsmann Stiftung 2008, S. 38). Die Krise der kommunalen Finanzen in der öffentlichen Diskussion ist primär die Krise der Kernhaushalte und damit der Kernverwaltungen.

\section{Quantitative Bedeutung kommunaler Wirtschaft}

Die Diskussion um die Zusammenhänge kommunaler Wirtschaft und Haushaltskonsolidierung bewegt sich meist zwischen Extremen. Sie ist einerseits auf die Frage des Verkaufs kommunaler Unternehmen oder Unternehmensanteile unter dem Schlagwort „Privatisierung“ reduziert (z. B. Candeias, Rilling, Weise 2008). Vertreter der privaten Wirtschaft kritisieren ein Ausufern der Kommunalwirtschaft mit rein fiskalischem Ziel (vgl. Caspari 2009). Die Stadtverwaltungen selbst fokussieren häufig auf die Stadtwerke als kommunale „Cash Cow“. Ein Teil der akademischen Diskussion ist primär auf die staatlichen Ebenen fokussiert (Schmidt 2011; Schmitt, Obinger 2010).

Eine zahlenbasierte Grundlage bietet die jährliche Gemeindefinanzstatistik des Statistischen Bundesamtes. Tabelle 1 weist einige zentrale Haushaltskennzahlen für die Jahre 1997 bis 2007 aus. Zahlen auf dieser Aggregationsebene lassen naturgemäß Fragen und die Wirkung verschiedener Einflussfaktoren offen und können unterschiedlich interpretiert werden. Grundsätzlich wird als Ausdruck der kommunalen Finanzkrise in der Summe der elf Haushaltsjahre ein negativer Finanzierungssaldo (7,896 Milliarden Euro) deutlich. Er bemisst sich im Durchschnitt zwar „nur“ auf 0,5 Prozent der kumulierten Gesamtausgaben, gewinnt jedoch Brisanz aus der regional unterschiedlichen Verteilung und den skizzierten Rechtsfolgen.

Mit Blick auf die wirtschaftliche Tätigkeit betragen die daraus resultierenden Einnahmen im Schnitt knapp sechs Prozent der bereinigten Gesamteinnahmen der Kommunen. Davon bilden die 
Konzessionsabgaben, die unabhängig der Trägerschaft der Unternehmen zu zahlen sind, mit rund der Hälfte den größten Anteil, Gewinnabführungen circa ein Fünftel. Die Bedeutung dieser Einnahmequelle muss in Bezug zu den Gesamteinnahmen gesehen werden, die langfristig steigen. Ihr Anteil bleibt über die Jahre auffallend konstant, so dass sich das verschiedentlich proklamierte Ausufern der öffentlichen Wirtschaft (vgl. Brandt, Fuest 2007) nebst Gewinnmaximierung im Durchschnitt aller Länder nicht erschließt.

Sehr viel bedeutender im Volumen sind hingegen die Gebühreneinnahmen, ebenso eine Quelle mit gewissem Gestaltungsspielraum der Kommunen und mit Berührungspunkten zur Kommunalwirtschaft. Im Zuge von Maßnahmen der Haushaltskonsolidierung ist die Erhöhung von vor allem Benutzungsgebühren stets einer der ersten, wenn auch politisch heiklen, Schritte. Er wird überdies von der Rechtsaufsicht gefordert. Vor diesem Hintergrund ist es mehr als überraschend, dass die Gebühreneinnahmen im Zeitverlauf sinken. Dies lässt sich aus der Auslagerung gebührenrechnender Einrichtungen erklären. Noch vor wenigen Jahrzehnten wurden klassische Aufgabenbereiche wie Ver- und Entsorgung innerhalb des Kernhaushaltes z. B. in Regiebetrieben abgebildet. Mit dem geschilderten Trend der Konzernbildung wurden diese Leistungen zunehmend verselbständigt oder formal privatisiert und finden sich nun in den Umsätzen der Unternehmen wieder. Wie erwähnt, sind diese im Verhältnis zum Kernhaushalt beständig gestiegen. Zurück bleiben die (Verwaltungs)gebühren, die sich nicht für eine Auslagerung eignen und kommunal wenig gestaltbar sind. Dies bedeutet jedoch nicht, dass diese verlagerten Gebühreneinnahmen analog auf der Seite der Einnahmen aus Wirtschaftstätigkeit verbucht werden. Sie „,verschwinden" hingegen zumindest teilweise in den Weiten des Konzernbundes.

Ein bemerkenswerter Punkt auf der Ausgabenseite sind die Personalkosten. Hier zeigt sich über die Jahre 1997 bis 2007 ein lediglich minimaler Zuwachs von vier Prozent, weit unter den Tarifanpassungen des öffentlichen Dienstes oder der Inflationsrate. Auch hieraus lassen sich zwei Thesen ableiten. Jede Kommune hat im Zuge der Haushaltskonsolidierung bei den Personalkosten als einem der größten und vermeintlich nicht direkt bürgerwirksamen Kostenblöcke angesetzt und erheblich Stellenzahlen reduziert. Hinter den relativ stabilen Lohnkosten verbergen sich daher vornehmlich sinkende Stellenzahlen in der Kernverwaltung. Die zweite Erkenntnis ist analog zur Verschiebung von Umsätzen die Verschiebung von Personal in die Beteiligungen.

Ein häufig nicht berücksichtigter Punkt auf der Ausgabenseite im Zusammenhang mit kommunalen Unternehmen ist die Frage der Zuschüsse an diese. Es sind eben nicht alle Beteiligungen profitabel und auch der Konzern in Gänze ist trotz Querverbund nicht in der Lage, kostendeckend zu wirtschaften. Eine Vielzahl Leistungen der Unternehmen muss aus dem Kernhaushalt, damit den allgemeinen Deckungsmitteln Steuern und Schlüsselzuweisungen, bezuschusst werden (z. B. Kultur, Soziales, Städtebau, Wirtschaftsförderung). Diese Zuschüsse belaufen sich auf circa die Hälfte der Erträge aus Wirtschaftstätigkeit. Wenn man so will, beträgt der Nettoertrag aus wirtschaftlicher Betätigung für den Kernhaushalt damit nur rund drei Prozent der Gesamteinnahmen. Auf eine wesentliche Subventionierung der Kernverwaltung durch die Unternehmen kann aus diesem Zahlungsstrom kaum geschlossen werden. ${ }^{4}$ Interessanterweise sind die Zuschüsse aus dem Kernhaushalt an die Unternehmen tendenziell gestiegen. Dies kann in umfangreichen formellen

4 Auch statistische Analysen dieser Zeitreihen ergeben keinen signifikanten Zusammenhang z. B. zwischen Einnahmen aus Wirtschaftstätigkeit, Beteiligungsveräußerung und dem Finanzierungssaldo. 
Privatisierungen nicht kostendeckender Leistungen begründet liegen (Truger 2008, S. 552). Über die formelle Privatisierung und Ausgliederung aus dem Kernhaushalt wird dieser Zahlungsstrom transparent.

\begin{tabular}{|l|c|c|c|c|c|c|c|}
\hline & $\begin{array}{c}\text { Perso- } \\
\text { nalkos- } \\
\text { ten }\end{array}$ & $\begin{array}{c}\text { Zuschüs- } \\
\text { se an Un- } \\
\text { terneh- } \\
\text { men }\end{array}$ & $\begin{array}{c}\text { Finanz. } \\
\text { Saldo }\end{array}$ & $\begin{array}{c}\text { bereinigte } \\
\text { Einnah- } \\
\text { men }\end{array}$ & $\begin{array}{c}\text { Gebüh- } \\
\text { renein- } \\
\text { nahmen }\end{array}$ & $\begin{array}{c}\text { Einnahmen aus } \\
\text { Wirtschaftstä- } \\
\text { tigkeit }\end{array}$ & $\begin{array}{c}\text { Anteil an } \\
\text { Einnah- } \\
\text { men }\end{array}$ \\
\hline 1997 & 38.980 & 3.029 & -2.836 & 140.848 & 18.159 & 7.446 & $5,3 \%$ \\
\hline 1998 & 38.710 & 3.467 & 2.155 & 144.658 & 17.533 & 7.688 & $5,3 \%$ \\
\hline 1999 & 39.190 & 3.431 & 2.182 & 145.923 & 17.201 & 7.899 & $5,4 \%$ \\
\hline 2000 & 39.550 & 3.708 & 1.934 & 148.009 & 17.116 & 7.707 & $5,2 \%$ \\
\hline 2001 & 39.388 & 4.262 & -4.089 & 144.243 & 16.681 & 8.250 & $5,7 \%$ \\
\hline 2002 & 40.000 & 5.211 & -3.661 & 146.304 & 16.193 & 10.073 & $6,9 \%$ \\
\hline 2003 & 40.547 & 5.256 & -8.395 & 141.524 & 16.304 & 8.979 & $6,3 \%$ \\
\hline 2004 & 40.485 & 5.724 & -3.879 & 146.195 & 16.251 & 8.746 & $6,0 \%$ \\
\hline 2005 & 40.746 & 4.884 & -2.237 & 150.945 & 15.956 & 8.838 & $5,9 \%$ \\
\hline 2006 & 40.673 & 4.827 & 2.755 & 160.066 & 16.090 & 8.950 & $5,6 \%$ \\
\hline 2007 & 40.598 & 4.983 & 8.175 & 169.713 & 16.107 & 9.649 & $5,7 \%$ \\
\hline Summe & $\mathbf{4 3 8 . 8 6 8}$ & $\mathbf{4 8 . 7 8 2}$ & $-\mathbf{7 . 8 9 6}$ & $\mathbf{1 . 6 3 8 . 4 2 8}$ & $\mathbf{1 8 3 . 5 9 1}$ & $\mathbf{9 4 . 2 2 5}$ & $\mathbf{5 , 8 \%}$ \\
\hline
\end{tabular}

Tabelle 1: Haushaltskennzahlen im Zeitverlauf, Angaben in Millionen Euro für Gemeinden und Gemeindeverbände

Quelle: Statistisches Bundesamt

Tabelle 2 widmet sich speziell den Finanzbewegungen bezüglich des Erwerbs und der Veräußerung von Beteiligungen, Letzteres als materielle Privatisierung bezeichnet und der eigentliche Kern der Privatisierungsdiskussion. Die Kommunen haben in der dargestellten Periode 1997 bis 2007 Veräußerungen vorgenommen. Die Veräußerungserlöse aus Beteiligungen summierten sich über diese elf Haushaltsjahre auf einen Betrag, der rund einem Prozent der summierten bereinigten Einnahmen entspricht. Inwieweit sich hierunter Komplettverkäufe von Unternehmen verbergen, ist aus den Zahlen nicht ersichtlich, da diese nicht erfasst werden. Es ist davon auszugehen, dass es sich weit überwiegend um anteilige Veräußerungen handelt, die nicht allein fiskalischen sondern auch unternehmensstrategischen Zwecken dienten. Es wird ebenso deutlich, dass es sich in Erwerb und Veräußerung von Beteiligungen um zwei parallel laufende Prozesse handelt, die von punktuellen Ausschlägen begleitet werden. Ein langfristiger Anstieg der VeräuBerungen ist nicht ersichtlich. Der Gesamtwert aller kommunalen Unternehmen ist (noch) nicht bekannt, sodass nicht hinterfragt werden kann, ob dieses Veräußerungsvolumen quantitativ relevant und bestandsgefährdend ist. Langfristig werden die Kommunen im Zuge der Doppik-Umstellung konsolidierte Konzernbilanzen aufstellen, die den Wert aller kommunalen Beteiligungen ausweisen.

Der Veräußerungserlös aus Beteiligungen relativiert sich in zweierlei Hinsicht. Der Bestand der Unternehmen befindet sich in einer gewissen (geringen) Fluktuation. So kommen neben der Ver- 
äußerung auch Neuerwerbungen vor, welche wiederum den Bestand erhöhen. Um die These des „Ausverkaufs“ kommunaler Unternehmen zu beurteilen, sind beide Transaktionsrichtungen zu saldieren. Der Verkaufssaldo entspricht summiert in der betrachteten Periode 0,3 Prozent der kumulierten Gesamteinnahmen. Ist die fiskalische Bedeutung der Beteiligungsveräußerung daher als gering zu bezeichnen? Ginge man davon aus, dass der Verkaufssaldo primär konsolidierenden Zielen für die Kernverwaltung dient, der ohne die Haushaltskrise nicht aufträte, so wäre die Anteilsveräußerung nicht unerheblich. Denn ohne sie betrüge der Finanzierungssaldo der Jahre 1997 bis 2007 nicht 7,896 Milliarden Euro sondern 12,617 Milliarden. In dieser Perspektive wäre mehr als ein Drittel des negativen Finanzierungssaldos über Anteilsveräußerungen gedeckt worden. Die Frage lässt sich jedoch nicht eindeutig beantworten, da keine repräsentativen Zahlen über konkrete Veräußerungsmotive vorliegen. Gleichwohl sind die wenigen spektakulären Großverkäufe (z. B. in Dresden, Düsseldorf) eher Ausreißer und vermitteln kein allgemeingültiges Bild.

Die Veräußerungserlöse der Kommunen relativieren sich in einer zweiten Perspektive, nämlich im Vergleich zu Bund und Ländern. Obgleich die staatlichen Unternehmen am Bestand öffentlicher Unternehmen lediglich rund 10\% ausmachen, entfielen im Zeitraum 1997 bis 2007 im Durchschnitt rund 89\% der Veräußerungserlöse auf Bund und Länder. Für diese Ebenen nahmen auch die Privatisierungserlöse an den Gesamteinnahmen ein wesentlich höheres Niveau ein, denn für die Kommunen. ${ }^{5}$ Materielle Privatisierungen (in Form von Anteilsverkäufen) waren somit eher ein Thema für Bund und Länder, während sich die Kommunen auf formelle Privatisierungen (Rechtsformänderungen) beschränkten.

Tabelle 2 zeigt im Vergleich den Verkaufssaldo aus unbeweglichem Vermögen (Immobilien). Hier handelt es sich tatsächlich um einen flächendeckenden Trend der Haushaltskonsolidierung (Naßmacher, Naßmacher 1999, S. 218; Bertelsmann Stiftung 2008, S. 72 ff.). Dessen fiskalische Relevanz ist um ein Vielfaches größer denn die Beteiligungsveräußerung.

5 Im Zeitraum 1997 bis 2005 deckte der Bund im Durchschnitt 2,96 Prozent seiner Einnahmen über Privatisierungserlöse, die westdeutschen Bundesländer 1,8 Prozent und die westdeutschen Kommunen 1,39 Prozent. Vgl. Zahlen aus Truger 2008. Im Jahr 1998 beliefen sich Veräußerungserlöse auf 4,96 Prozent der Bundeseinnahmen. 


\begin{tabular}{|l|c|c|c|c|c|}
\hline & $\begin{array}{c}\text { bereinigte } \\
\text { Einnah- } \\
\text { men }\end{array}$ & $\begin{array}{c}\text { Veräußerung von } \\
\text { Beteiligungen }\end{array}$ & $\begin{array}{c}\text { Erwerb von } \\
\text { Beteiligungen }\end{array}$ & $\begin{array}{c}\text { Verkaufssaldo } \\
\text { Beteiligungen }\end{array}$ & $\begin{array}{c}\text { Verkaufssaldo } \\
\text { unbewegliches } \\
\text { Vermögen }\end{array}$ \\
\hline 1997 & 140.848 & 835 & 1.161 & -326 & 3.038 \\
\hline 1998 & 144.658 & 1.722 & 1.064 & 658 & 2.885 \\
\hline 1999 & 145.923 & 1.090 & 1.168 & -78 & 2.673 \\
\hline 2000 & 148.009 & 1.687 & 1.155 & 532 & 1.986 \\
\hline 2001 & 144.243 & 1.855 & 1.962 & -107 & 1.514 \\
\hline 2002 & 146.304 & 2.425 & 925 & 1.500 & 911 \\
\hline 2003 & 141.524 & 1.718 & 936 & 782 & 2.138 \\
\hline 2004 & 146.195 & 1.415 & 843 & 572 & 1.998 \\
\hline 2005 & 150.945 & 1.333 & 1.110 & 223 & 1.751 \\
\hline 2006 & 160.066 & 1.932 & 1.085 & 847 & 1.166 \\
\hline 2007 & 169.713 & 1.215 & 1.097 & 118 & $\mathbf{2 2 . 4 0 4}$ \\
\hline Summe & $\mathbf{1 . 6 3 8 . 4 2 8}$ & $\mathbf{1 7 . 2 2 7}$ & $\mathbf{1 2 . 5 0 6}$ & $\mathbf{4 . 7 2 1}$ & \\
\hline
\end{tabular}

Tabelle 2: Erwerb und Veräußerung von Beteiligungen im Zeitverlauf, Angaben in Millionen Euro für Gemeinden und Gemeindeverbände

Quelle: Statistisches Bundesamt

\section{Auslagerungen und ihr Konsolidierungsbeitrag}

\section{Formen von Auslagerungen}

Nichtsdestotrotz können die Zahlen der Gemeindefinanzstatistik kein vollständiges Bild der Relevanz kommunaler Wirtschaft im Rahmen der Haushaltskonsolidierung aufzeigen. Den Kommunen steht ein weites Spektrum organisatorischer Varianten offen, deren Wahl auch fiskalisch begründet ist und deren Haushaltseffekte aus der Finanzstatistik nicht hervortreten.

Die Organisationshoheit der Kommunen stellt es ihnen frei, in welcher Rechts- oder Organisationsform Aufgaben erfüllt werden. Die geschilderten Umfeldfaktoren wie Leitbildwandel, EULiberalisierung, Unzufriedenheit mit der tradierten Verwaltung oder Haushaltsdruck führten zu vielfältigen Auslagerungen kommunaler Aufgaben aus der Kernverwaltung in öffentliche oder private Rechtsformen (Greiling 2011, S. 3;).

In den vergangenen zwanzig Jahren nutzten die Kommunen diese Formenvielfalt vermehrt, um diesen Umfeldfaktoren zu begegnen. Das Resultat ist eine starke Zunahme der Heterogenität der Organisationsstrukturen, die zumindest auf großstädtischer Ebene häufig kaum noch überschaubar sind. Der ,institutionelle Rahmen der Kommunalverwaltung“ hat sich wesentlich geändert, womit auch die Anforderungen an die kommunale Beteiligungssteuerung stiegen (Reichard 2004, S. 55).

Innerhalb des Begriffes Auslagerung unterscheiden wir zwischen Verselbständigung und Privatisierung. Verselbständigung bedeutet die Auslagerung an einen öffentlich-rechtlichen Träger. 
Der Begriff Privatisierung ist hingegen vage, wird unterschiedlich abgegrenzt und verliert ,in der Heterogenität der abzubildenden Geschehnisse schnell jegliche Konturen“ (vgl. Gusy 1998; Budäus 2000; Schubert 2010, S. 75). Allgemein kann unter ihm die Beteiligung Privater an der öffentlichen Aufgabenerstellung gefasst werden. In diesem Beitrag unterscheiden wir vier Formen (vgl. Tabelle 3). Diese Organisationsformen stellen ein Kontinuum dar, beinhalten gleichsam ein Spektrum der Entkommunalisierung und eröffnen Spielräume der Haushaltsentlastung.

\begin{tabular}{|c|c|c|}
\hline & Kernverwaltung & $\begin{array}{l}\text { Optimierung der Aufbau- und Ablauforganisation der für die Aufgabe zustän- } \\
\text { digen Organisationseinheit } \\
\text { - Reduzierung der Erstellungskosten }\end{array}$ \\
\hline \multirow{6}{*}{ 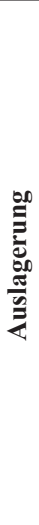 } & $\begin{array}{l}\text { Verselbständi- } \\
\text { gung }\end{array}$ & $\begin{array}{l}\text { Aufgabenübertragung auf öffentlich-rechtliche Rechtsform mit separater Or- } \\
\text { ganisation und Haushalt, z. B. Eigenbetrieb, Anstalt, Zweckverband } \\
\text { - Optimierung der Zuschüsse und Gewinnabführungen }\end{array}$ \\
\hline & Privatisierung & \\
\hline & Formell & $\begin{array}{l}\text { Überführung der Aufgabe in private Rechtsform im vollständigen Eigentum der } \\
\text { Kommune (Organisationsprivatisierung) z. B. GmbH, selten AG } \\
\text { - Optimierung Zuschüsse und Gewinnabführungen }\end{array}$ \\
\hline & Funktional & $\begin{array}{l}\text { Übertragung einzelner Prozesse an Private } \\
\text { - Reduzierung der Preise ggü. Eigenerstellungskosten }\end{array}$ \\
\hline & $\begin{array}{l}\text { Materielle } \\
\text { Teilprivatisierung }\end{array}$ & $\begin{array}{l}\text { Verkauf von Gesellschaftsanteilen an Private } \\
\text { - Außerordentliche Einnahmen } \\
\text { - Durch Integration privaten Know-Hows geringere Produktionskosten }\end{array}$ \\
\hline & $\begin{array}{l}\text { Materielle } \\
\text { Vollprivatisierung }\end{array}$ & $\begin{array}{l}\text { Verkauf aller Gesellschaftsanteile an Private } \\
\text { - Außerordentliche Einnahmen }\end{array}$ \\
\hline
\end{tabular}

Tabelle 3: Organisationsformen kommunaler Aufgabenwahrnehmung und mögliche Haushaltsentlastung

Quelle: Eigene Darstellung

Eine formelle Privatisierung bedeutet lediglich einen Rechtsformwechsel, eine funktionale Privatisierung die Übertragung von Teilprozessen einer Leistung. Hier bestehen die Erwartungen schlicht in wirtschaftlicheren Abläufen und somit geringeren laufenden Kosten. Die Hoheit über die Leistung als Ganzes verbleibt hingegen in der Kommune. Eine materielle Privatisierung bedeutet demgegenüber die Aufgabe alleiniger Zuständigkeit und Entscheidungen und damit gleichsam wachsende Steuerungsansprüche. Vollständige materielle Privatisierungen sind äußerst selten. Dies erklärt sich nicht allein aus der Politisierung der Entscheidungsfindung sondern auch daraus, dass wenige Verwaltungsbereiche vorstellbar sind, in denen die kommunale Aufgabe vollends entfallen kann und nicht zumindest eine kommunale Gewährleistungspflicht besteht. Tatsächlich bleibt, zumindest aus Sicht der Bürger, eine Auffangzuständigkeit der Kommune erhalten, welche die wirtschaftlichen Privatisierungsziele konterkariert.

\section{Motive für Auslagerungen}

Wie erwähnt, ist der Trend zur Auslagerung in engem Zusammenhang zur Haushaltskrise der Kommunen, zum NSM, der Unzufriedenheit mit der Kernverwaltung und zur EU Liberalisie- 
rungspolitik zu sehen. ${ }^{6}$ Der Einfluss dieser vier übergreifenden Trends muss jedoch für bestimmte Sektoren der kommunalen Wirtschaft unterschiedlich beantwortet werden. Kann die Marktliberalisierung durchaus als dominant im Sektor Strom und Gas gesehen werden (vgl. Le Nestour 2001), so vermag diese Erklärung für nicht liberalisierte Märkte nicht zu überzeugen.

Die Auslagerungsmotive aus Sicht der einzelnen Kommune mögen vielschichtig und nachvollziehbar sein. Ein häufig genanntes Motiv ist die Erhöhung der Flexibilität in der Leistungserbringung durch die „Befreiung“ aus den engen Grenzen des öffentlichen Rechts und damit auch eine leichtere Verwaltungsmodernisierung, die in der Kernverwaltung zunehmend an Grenzen stieß (Reichard 2004, S. 58; Bogumil et al. 2010, S. 61 f.; Eichhorn 1997). Hinzu traten die Erwartung höherer Leistungsqualität, geringeren politischen Einflusses, Einbindung privaten Kapitals und Fachwissens, Größenvorteile durch Kooperation oder besserer Markterschließung (Schwarting 2001, S. 304; Trappe 2003, S. 39 f.; Le Nestour 2001). Sicher nicht zu vernachlässigen sind Effekte des ,,institutionellen Isomorphismus“ der Kommunen, gewissermaßen ein Herdentrieb der Organisationspolitik (DiMaggio, Powell 1983, S. 150 ff.). Gleichsam galten im Kontext des Leitbildes der neunziger Jahre private Rechtsformen schlicht als modern und es wurde ihnen eine per se höhere Wirtschaftlichkeit unterstellt. Einige Autoren sehen Auslagerungen auch als Indiz einer generellen Überforderung des Staates (Gusy 1998, S. 330; Hardrath 2003, S. 53).

Es ist somit sicher verkürzt, Auslagerungen allein auf die Haushaltskrise der Kommunen zurückzuführen. In der Erhöhung der Wirtschaftlichkeit der Aufgabenerfüllung und damit einer Haushaltsentlastung, wird jedoch der „,mit Abstand wichtigste Grund“ gesehen (Bogumil et al. 2010, S. 60). All diese Aspekte lassen den Eindruck einer gewissen Ohnmacht der lokalen Entscheidungsträger angesichts vielfältiger Herausforderungen des Umfeldes entstehen, als deren Patentlösung Auslagerungen erkannt wurden. Es ist anzunehmen, dass fiskalische Gesichtspunkte bei fast jeder Auslagerung im Raum standen (Richter Edeling 2011, S. 146 f.; Reidenbach 2009). Sie können das empirische Phänomen jedoch nicht abschließend erklären. Veräußerungen von Anteilen oder Unternehmen in Gänze zum Abbau von Haushaltsdefiziten waren sicher nicht die Regel, gleichwohl bis Ende der neunziger Jahre akzeptierte Praxis (Kurth 1999; Richter 1995; Geißler 2011 b, S. 216 ff.). ${ }^{7}$

Wenn Auslagerungen als ein Element der Staatsmodernisierung betrachtet werden (vgl. Greiling 2011), ergäben sich zwei Erkenntnisse: Sie stünden einerseits als Beleg für begrenzte Modernisierungspotenziale innerhalb tradierter Formen der Kernverwaltung, gleichzeitig aber auch für die Notwendigkeit intensiverer privat-öffentlicher Kooperationen in der Erbringung öffentlicher Leistungen. Diese Zunahme der Kooperation ist tatsächlich beobachtbar, wenn auch unterschiedlich stark in den Sektoren (Bogumil et al. 2010, S. 57). Auch diese Kooperation ist getrieben von der Erwartung höherer Wirtschaftlichkeit (Strünck, Heinze 2005, S. 123).

Demgegenüber besteht ein direkter quantitativ messbarer Zusammenhang zur EU-Liberalisierung, abzulesen aus unterschiedlichen Auslagerungsgraden zwischen Sektoren. So werden noch nicht liberalisierte Sektoren (z. B. Abwasser) überwiegend in öffentlicher Rechtsform geführt (Bogumil et al. 2010, S. 57). Im früh liberalisierten Strommarkt finden sich nicht allein fast aus-

6 Die Beurteilung der Wirtschaftlichkeit von Auslagerungen steht in engem Zusammenhang zu steuerrechtlichen Fragen. Diese sind nicht Inhalt dieses Beitrages.

7 Es handelt sich hierbei um eine weit zurückreichende Debatte. Vgl. Wissenschaftlicher Beirat der Gesellschaft für Öffentliche Wirtschaft und Gemeinwirtschaft 1979 oder van Loesch 1997. 
schließlich private Rechtsformen, sondern auch ein wesentlich höherer Anteil gemischtwirtschaftlicher Unternehmen (Trappe 2003, S. 29 f.). In diesem Sektor hat der schlagartig eingesetzte Wettbewerb die Marktstruktur nachhaltig verändert. Die allgemeine Zunahme ausgelagerter kommunaler Leistungen wird somit begleitet von einer sektoralen Heterogenität (Bogumil, Holtkamp 2006, S. 93 ff.; Richter, Kilian, Trapp 2006). Diese Erkenntnis bestätigt die wesentliche Rolle der EU im Wandel kommunaler Organisationsstrukturen (Deckwirth 2008, S. 534-537).

Der bundesdeutsche Gesetzgeber schuf in den neunziger Jahren die rechtlichen Erleichterungen für Auslagerungen (Hardrath 2003, S. 61). Gleichwohl bedurften sie stets einer eigenständigen politischen Entscheidung in den Kommunen, welche sich hiermit gewissermaßen „freiwillig selbst entmachteten".

Worin die konkreten Motive der einzelnen Kommunen für diese Entscheidungen bestanden, ist hingegen meist nicht bekannt, da die Beteiligungsberichte hierzu mehrheitlich keine Aussage treffen (Richter, Kilian, Trapp 2006, S. 124). Ebenso waren Auslagerungsentscheidungen im politischen Raum selten ein „Resultat methodischer Überlegungen und systematischer Leistungstiefenentscheidungen“ (Mezger 2007, S. 54). Angesichts nicht definierter Ziele ist es schwierig, den Erfolg der Auslagerungen zu beurteilen.

\section{Haushaltsentlastende Effekte}

Sofern die Haushaltskrise ein zentraler Auslöser von Auslagerungen in Kommunen war, so müssen diese, zumindest in der Erwartungshaltung, mit entlastenden Effekten für die Haushalte der Kommunen verbunden gewesen sein. In diesem Abschnitt wird über die Gemeindefinanzstatistik hinaus die Frage aufgeworfen, worin diese bestehen können (vgl. Abbildung 3). 


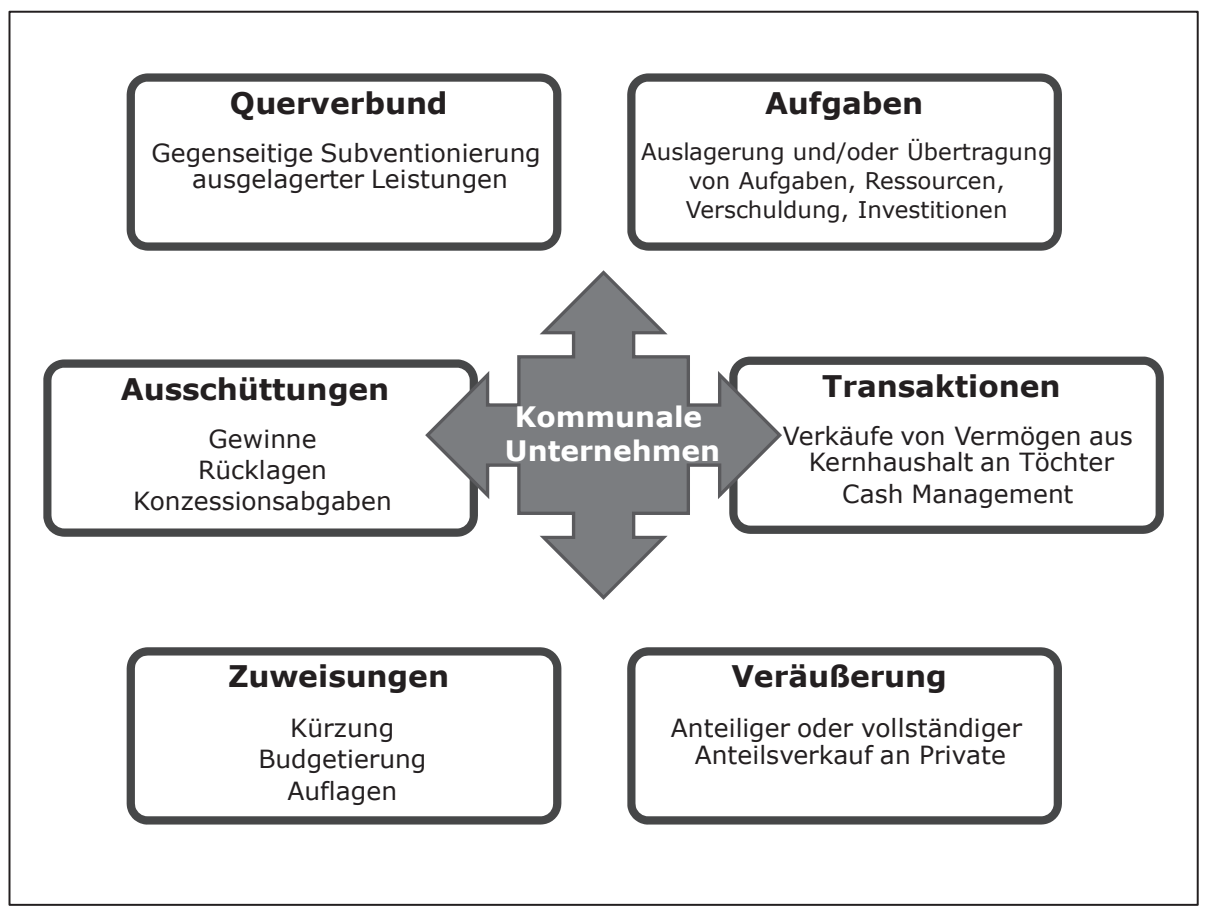

Abbildung 3: Haushaltsentlastende Effekte aus kommunalen Unternehmen

Quelle: Eigene Darstellung

Mit der Auslagerung kommunaler Leistungen aus der Kernverwaltung ist parallel auch die Auslagerung der betreffenden Einnahmen und Ausgaben aus dem Kernhaushalt verbunden, da die neuen Rechts- und Organisationsformen eigene Wirtschaftspläne aufstellen. Im Regelfall liegt jedoch weiterhin eine Verbindung über zumindest eine Zahlungsbewegung vor, denn je nach Kostendeckungsgrad aus eigenen Einnahmen wird die Auslagerung entweder aus dem Kernhaushalt bezuschusst oder führt Erträge an diesen ab. Sofern die Erwartung höherer Wirtschaftlichkeit der Auslagerung, eines der Hauptmotive, aufgeht, besteht der finanzwirtschaftliche Effekt für den Kernhaushalt entweder in einer Reduzierung der Zuschüsse oder höheren Erträgen aus den Auslagerungen.

Aus haushalterischer Sicht ist eine Auslagerung automatisch budgetiert. Dieser effektive Ansatz der Haushaltskonsolidierung ist innerhalb der Kernverwaltung sehr viel schwieriger umzusetzen (Bogumil et al. 2007, S. 51 ff.). Die fiskalische Steuerung wird über die Begrenzung auf lediglich einen Zahlungsstrom erheblich verbessert, da Rationalisierungsimpulse über dessen Gestaltung direkt vermittelt werden. Gleichzeitig kann diese Zahlungsbewegung seitens der Eigentümerin mit Kennzahlen und Zielen verbunden werden. Aus der Entstehung der Beteiligungsverwaltung in der Kämmerei und dem Fokus auf Wirtschaftlichkeit kann eine zu starke Konzentration auf rein finanzielle Vorgaben resultieren. In Phasen der Haushaltskonsolidierung kommt es ebenso häufig zu willkürlichen Festsetzungen dieser Größen, um Auflagen der Rechtsaufsicht hinsicht- 
lich von Konsolidierungsvolumina zu erfüllen. Eine weitere direkte Einnahme ähnlich den Gewinnabführungen sind die Konzessionsabgaben, die auch kommunale Unternehmen an ihre Eigentümer zu entrichten haben. Deren Bemessung ist in der maximalen Höhe reguliert und nicht direkt abhängig vom Grad der Auslagerung.

Mit der Auslagerung von Leistungen aus dem Kernhaushalt geht neben der Auslagerung von Umsätzen und Personal häufig auch die Auslagerung von Verschuldung einher. Die Strategie buchhalterischer Entschuldung ist zumindest ein willkommener Nebeneffekt (Richter, Kilian, Trapp 2006, S. 123). Tatsächlich steigt der Anteil der ausgelagerten Verschuldung an der Gesamtverschuldung der Kommune seit Jahren beständig an und erreichte 2007 im Durchschnitt bereits über 50 Prozent (vgl. Bertelmann Stiftung 2008). Mit den Aufgaben können auch Investitionen ausgelagert werden, die über den Kernhaushalt für defizitäre Kommunen nicht mehr möglich sind. In der Folge werden sie von der Rechtsaufsicht unbehelligt durch das Unternehmen erbracht.

Eine komplexere Entwicklungsstufe kommunaler Auslagerungen ist der steuerliche Querverbund. Er dient dazu, Synergien und Skaleneffekte zu erwirtschaften, aber auch Gewinne eines ertragsstarken Unternehmens mit den Verlusten eines ertragsschwachen in einem gemeinsamen Konzern zu verrechnen und in der Summe die Steuerlast des Verbundes zu mindern. Obgleich die Möglichkeiten derlei Verbünde durch die EU auf bestimmte Branchen begrenzt wurden, sind deren finanzielle Effekte hoch. Dass es nicht möglich ist, ihr Volumen exakt zu benennen, weist auf den wesentlichen Nachteil des Querverbundes hin. Er liegt in der Agglomeration und finanzwirtschaftlichen Verflechtung von Unternehmen und Leistungen, so dass einzeln entstehende Kosten und Erträge nicht mehr ersichtlich sind. Nichtsdestotrotz sind steuerliche Querverbünde auch in der Privatwirtschaft üblich. Für die Kommunen sind sie ein historisch gewachsenes Instrument, um z. B. den ÖPNV zu finanzieren.

Die Veräußerung von Unternehmensanteilen ist eine Option, kurzfristig spürbare Beträge zu erwirtschaften. Diesbezüglich ist in den vergangenen zwanzig Jahren ein Paradigmenwechsel zu beobachten. Waren Anteilsveräußerungen Anfang der neunziger Jahre noch en vogue, hat sich das Meinungsbild nach dem Jahr 2000 gewendet (Theuvsen, Zschache 2011). Solche Transaktionen standen eher selten einzig unter dem Ziel der Haushaltskonsolidierung (Richter, Kilian, Trapp 2006, S. 117). Wesentliche Veräußerungen sind politisch nicht mehr durchsetzbar und gelten auch haushalterisch als nicht zweckmäßig im Vergleich zu den organisatorischen Optionen in großen Konzernen oder schlicht den laufenden Gewinnabführungen. Dennoch können Anteilsverkäufe zum Zweck der Haushaltskonsolidierung sinnvoll sein, wenn die Einnahmen zum Schuldenabbau verwendet werden und über die Reduzierung der Zinslast das strukturelle Defizit nachhaltig ausgeglichen werden kann (vgl. Geißler 2011 a). Die Zahl der von vollständiger oder anteiliger Privatisierung betroffenen Unternehmen wird statistisch nicht erfasst und ist daher nicht bekannt. Grundsätzlich ist davon auszugehen, dass mit zunehmender Größe der Stadt solche Transaktionen zunahmen. Dies wird erklärbar aus dem schlicht größeren Potential von Unternehmen in Großstädten und der größeren Attraktivität dieser für private Investoren. Auch für die Zukunft sind anteilige Veräußerungen kommunaler Unternehmen in einem Teil der Kommunen als Reaktion auf Einnahmerückgänge zu erwarten.

Über Auslagerungen eröffnen sich Möglichkeiten kreativer Buchhaltungslösungen zugunsten des Kernhaushaltes. So war es den Kommunen möglich, z. B. Immobilien an eigene Unternehmen zu 
veräußern und diese Erträge im Kernhaushalt zum Ausgleich von Defiziten zu verbuchen. Der Vorteil besteht darin, dass Eigentum faktisch nicht verlorengeht und dennoch außerordentliche Erträge erwirtschaftet werden. Diese Option der Ersatzdeckung wird mittelfristig im Zuge von Konzernabschlüssen der Stadt und ihrer Beteiligungen ausgeschlossen sein.

Die entlastenden Effekte kommunaler Unternehmen für die Kernhaushalte gehen über rein fiskalische Zahlungsströme hinaus. Tatsächlich ist es historisch gewachsener Zweck der Unternehmen, politisch gesetzte Aufgaben zu erfüllen. Demzufolge kann der Stadtrat als Eigentümer den Unternehmen quasi jedwede kommunale Aufgabe übertragen, darunter eben auch solche, die innerhalb der Kernverwaltung z. B. aus finanziellen Engpässen und strenger Kommunalaufsicht nicht umsetzbar sind. Kostenträchtige Leistungen können an Unternehmen übertragen werden, welche sie quasi aus den Erträgen ihrer originären Leistungen mitfinanzieren. Auslagerungen erhöhen in einer positiven Betrachtung die Spielräume der Reorganisation und Finanzierung kommunaler Leistungen in einem Spektrum verschiedener Träger und Finanzierungsquellen.

Diese finanzwirtschaftlichen Effekte beruhen jedoch auch auf einer empirischen Paradoxie der Kommunalaufsicht. Denn obgleich diese die gesamte Haushaltssituation der Kommune überwachen soll und ihr Wirtschaftspläne der Auslagerungen vorliegen (Gern 2003, S. 459 ff.), reduziert sie ihre Analysen meist auf den Kernhaushalt. Finanzvorgänge, Defizite, Risiken oder Verschuldung in den Auslagerungen blieben ihr häufig verborgen. Ebenso beziehen sich die entsprechenden fiskalischen Regeln der Gemeindeordnungen bzw. weitergehender Rechtsnormen in der Feststellung einer gefährdenden Haushaltslage auf den Saldo des Kernhaushaltes (Geißler 2009, S. 9 ff.).

Schlussendlich bestehen eine Reihe Möglichkeiten, über Auslagerungen die Situation des Kernhaushaltes zu verbessern. Dem stehen jedoch auch Nachteile gegenüber. Der Kernhaushalt schrumpft im Umfang seiner Haushaltsstellen, seiner Umsätze, des Volumens, der Investitionen und Stellenzahlen, die nunmehr im ausgelagerten Wirtschaftsplänen geführt werden. Über Auslagerungen verringert sich der Handlungsspielraum im Kernhaushalt. Er läuft langfristig Gefahr, nur mehr einzelne Zahlungsströme und solche Leistungen zu beinhalten, die nicht auslagerungsfähig sind. Die Aussagekraft sinkt, was seiner Rolle als politischem Steuerungsinstrument widerspricht und ebenso die Beurteilung der realen Finanzlage nahezu unmöglich macht, solange kein konsolidierter Gesamtabschluss vorliegt. Die Gefahren liegen in einer Verlagerung kommunaler Verschuldung und dem unbeobachtetem Anwachsen von Risiken, für welche die Kommune letztlich haftet, die jedoch nur mehr eingeschränkt steuerbar sind.

Ebenso ist es zumindest diskussionswürdig, einen wachsenden Teil öffentlichen Aufgaben über Entgelte der Ver- und Entsorgungsdienste zu finanzieren. Gleichwohl waren und sind die Kommunen angesichts der Haushaltskrise und sehr begrenzter lokaler Einnahmehoheiten gezwungen, die Potentiale der Unternehmungen zu nutzen und dabei innovative Finanzierungswege zu erschließen.

Inwieweit die Erwartungen an eine höhere Wirtschaftlichkeit ausgelagerter Unternehmen sich erfüllten, ist strittig. Dem beobachtbaren Erfolg kommunaler Unternehmen auf liberalisierten Märkten stehen kritische Einschätzungen gegenüber (vgl. Greiling 2011; Mühlenlamp 2001). Sofern Auslagerungen tatsächlich effizienter arbeiten, begünstigen sie das Ziel, die Wirtschaftlichkeit der Gesamtverwaltung zu steigern, da ein wachsender Anteil in ihnen erbracht wird. 


\section{Rekommunalisierung}

Seit einigen Jahren häufen sich Berichte über die Rekommunalisierung von Leistungen und Unternehmen (z. B. Röber 2009; Engartner 2009; Bogumil et al. 2010; Scholle 2010; Lenk, Rottmann, Albrecht 2011 b). Der Begriff verhält sich im Grunde spiegelbildlich zu dem der Auslagerung. Vergleichbar vielfältig wird er interpretiert. Gemeinhin wird hierunter verstanden, Leistungen oder Unternehmensanteile, die an private Träger übertragen wurden, wieder in den kommunalen Konzern zu integrieren. Tabelle 4 zeigt Optionen auf, die als Rekommunalisierung verstanden werden.

\begin{tabular}{|l|l|}
\hline 1 & Umwandlung privater Rechtsform in öffentliche Rechtsform \\
\hline 2 & Rückkauf veräußerter Unternehmensanteile \\
\hline 3 & Rückkauf vollständig veräußerter Unternehmen \\
\hline 4 & Reintegration einzelner an private Träger übertragener Prozesse (Insourcing) \\
\hline 5 & Neugründung kommunaler Unternehmen \\
\hline 6 & Nichtverlängerung von Konzessionen an private Träger \\
\hline 7 & Pacht privater Netze durch die Kommune \\
\hline 8 & Integration von Leitungsnetzen in bestehende kommunale Unternehmen \\
\hline
\end{tabular}

\section{Tabelle 4: Varianten der Rekommunalisierung}

Quelle: Eigene Darstellung

Für diese Entscheidungen können ähnliche externe Trends verantwortlich gemacht werden, wie ehedem für die Phase der Auslagerungen in den neunziger Jahren (Abbildung 4). Nach vielen Jahren der Unsicherheit über die Zukunft kommunaler Wirtschaft im Zuge der EU Liberalisierung, hat diese einen Stand erreicht, an dem weitere Marktöffnungen nicht zu erwarten sind bzw. nicht mehr als Gefahr gesehen werden. Ein wesentlicher Unsicherheitsfaktor ist damit geschwunden. Gleichzeitig verlief die Marktöffnung in den betroffenen Sektoren wider Erwarten erfolgreich für die kommunalen Unternehmen. Diese agieren, der Intention ihrer Auslagerung folgend, wirtschaftlich und versuchen, den gegebenen Rechtsrahmen bestmöglich zu nutzen.

Das dem NSM in Teilen zu Grunde liegende Leitbild eines schlanken, auf Kernfunktionen reduzierten Staates hat sich als nicht zweckmäßig erwiesen (vgl. Wegrich 2010). Im Gegenteil rückten die Nachteile reduzierter Kernverwaltungen für die Steuerung ausgelagerter Einheiten als auch die Vorteile kommunaler Unternehmen in das Bewusstsein der Lokalpolitik. Das aktuelle Leitbild betont das erstarkte Selbstbewusstsein der Kommunalverwaltung, die im Interesse der Bürger und öffentlicher Dienste das ihr eröffnete Formenspektrum nutzt und stringent steuert.

Die Haushaltskrise hat die Kommunen nicht verlassen. Sie hat vielmehr zugenommen und ist zu einem beständigen dauerhaften Begleiter kommunaler Politik geworden. Angesichts der im vorherigen Kapitel aufgezeigten Varianten der Beteiligung kommunaler Unternehmen im Umgang mit der Haushaltskrise, sind die Kommunen nunmehr bestrebt, ihr Portfolio auszuweiten, um diese Konsolidierungsvarianten zu maximieren. Auch in Bezug zur Haushaltskrise hat ein Bewusstseinswandel stattgefunden. Die Unternehmen werden nicht länger als Belastung oder Risiko gesehen, sondern als Potenzial kreativer Gestaltung. Umso mehr sich die kommunale Wirtschaft als 
Strategie der Haushaltskonsolidierung erwies, umso mehr wurde die Auslagerungsstrategie der neunziger Jahre hinterfragt.

Der Bewusstseinswandel gegenüber öffentlichen Diensten und öffentlicher Trägerschaft beschränkte sich nicht allein auf die lokalen Entscheidungsträger sondern erreichte auch die Bürgerschaft als Ganzes. Umfragen zeigen, dass die Mehrheit der Bevölkerung die Versorgung über kommunale Träger präferiert. Nur eine Minderheit der Bevölkerung befürwortet weitere Privatisierungen. ${ }^{8}$ Das Interesse der Bürger an öffentlichen Leistungen ist angesichts des negativen Bildes von Privatisierungen gestiegen. Es wird begleitet von stärkeren Beteiligungsrechten in den Kommunen, die weitere Privatisierungen in vielen Fällen verhindert haben (z. B. Leipzig, Freiburg). Mit den Bürgern ist ein neuer Akteur der Debatte um Privatisierung und Rekommunalisierung beigetreten. Diesen Entwicklungen liegt jedoch auch die Gefahr (partei)politischer Instrumentalisierungen anheim, welche Ängste der Bevölkerung ausnutzt und durchaus sinnvolle Reorganisationen verhindert.

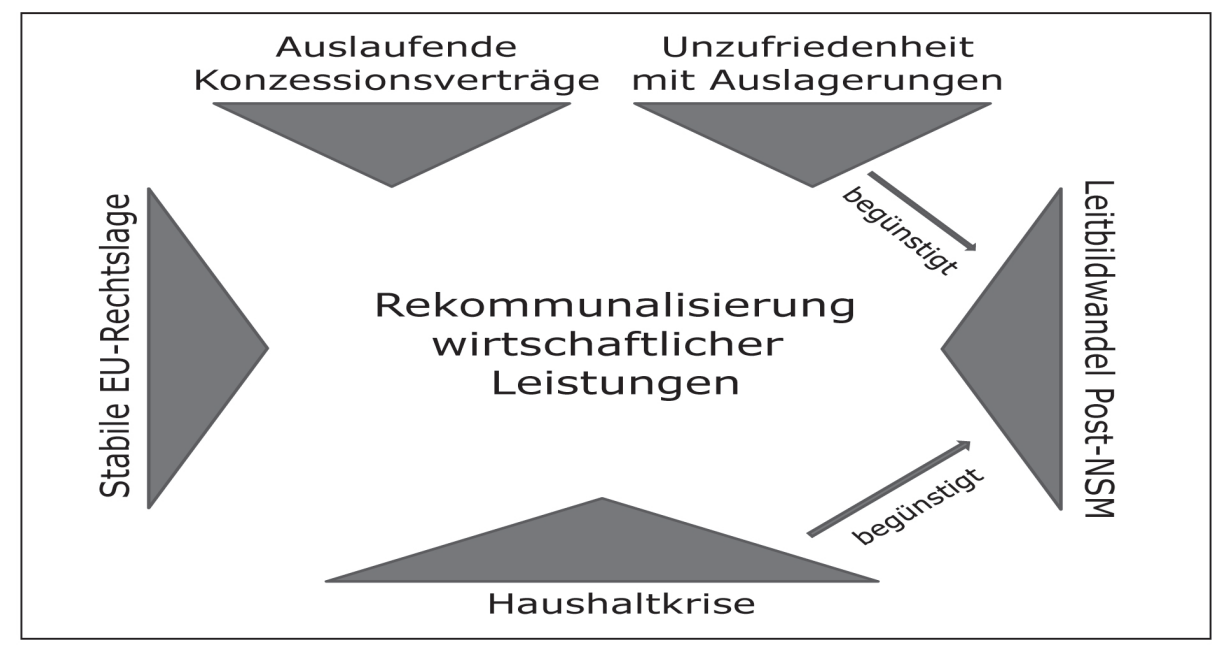

Abbildung 4: Treiber der Rekommunalisierung

Quelle: Eigene Darstellung

Die konkreten Motive der Kommunen, ausgelagerte Leistungen zu rekommunalisieren, sind ebenso vielfältig, wie jene, die ehedem zur Auslagerung führten (vgl. Lenk, Rottmann, Albrecht 2011). An die Stelle der früheren Unzufriedenheit mit der Kernverwaltung rückt die Unzufriedenheit mit privatisierten Leistungen. Diesbezügliche Erhebungen zeigen jedoch, dass die Erfahrungen der Städte nicht so negativ oder einheitlich sind, wie von Kritikern der Auslagerung oder Privatisierung unterstellt (Bogumil et al. 2007, S. 75; Lenk, Rottmann, Albrecht 2011 a; Röber 2009). Es scheint sich im Zuge der Planungen und Absichtserklärungen zur Rekommunalisierung

8 Vgl. Verband kommunaler Unternehmen 2010, S. 12.

$70 \%$ der Befragten lehnen die Privatisierung von Stadtwerken ab. 81\% der Befragten bringen Stadtwerken Vertrauen entgegen (zum Vergleich: Gewerkschaften 45\%, Großunternehmen 26\%). Vgl. Homepage VKU / Statistiken am 18.8.2011. 
um keinen übergreifenden Trend zu handeln, welcher auch nicht primär in enttäuschten Erfahrungen begründet liegt (vgl. Lenk, Rottmann, Albrecht 2011 b). ${ }^{9}$

Der wichtigste Auslöser der aktuellen Rekommunalisierungsdiskussion, welche sich zukünftig erst noch in Entscheidungen wandeln muss, ist vielmehr im mittelfristigen Auslaufen der Mehrheit der Konzessionsverträge der Strom- und Gasversorgung zu vermuten (Bogumil et al. 2010, S. 84). ${ }^{10}$ Hier handelt es sich um zwei Sektoren mit der am weitesten fortgeschrittenen Privatisierung. Für die Kommunen öffnet sich ein „Gelegenheitsfenster“, lukrative Leistungen wieder selbst zu erbringen und die in Kapitel 5.3 beschriebenen Haushaltseffekte zu nutzen. So zielt auch die öffentliche Argumentation klar auf diese wirtschaftlichen Vorteile (vgl. Verband Kommunaler Unternehmen 2010).

Dabei ist jedoch zu berücksichtigen, dass sich die Wirtschaftlichkeit von Rekommunalisierungen ebenso wenig per se einstellt wie die früherer Auslagerungen. Um die intendierten Effekte für den Haushalt zu erreichen, bedarf es zuvorderst der erfolgreichen Umsetzung eines rechtlich komplexen Prozesses, im Anschluss einer klugen Beteiligungssteuerung und guter Unternehmensführung (Röber 2009, S. 87). Trotz Rekommunalisierung besteht der Druck zur Ökonomisierung als Folge des Wettbewerbs fort. Auch die Fragmentierung des Gesamthaushalts oder die Heterogenität der Organisation nehmen nicht ab. Im Gegenteil erhöhen Rekommunalisierungen im Regelfall den Umfang der ausgelagerten Leistungen gegenüber dem Kernhaushalt, dessen Bedeutung in Beschäftigung, Umsatz, Investition und Verschuldung weiter sinkt. Es besteht die Gefahr, dass einige Kommunen mit diesen Herausforderungen überfordert sein werden und mittelfristig Rekommunalisierungen auch scheitern werden. Sie ist ebenso wenig ein Allheilmittel für die (Haushalts)nöte der Kommunen, wie es Auslagerungen sein konnten.

\section{Fazit}

Ziel dieses Beitrags ist es, Licht auf ein in der Praxis bedeutendes, wissenschaftlich jedoch wenig reflektiertes Thema zu werfen: die Bedeutung kommunaler Unternehmen in der Haushaltskonsolidierung. Ungeachtet vielfältiger vertiefender Fragen lassen sich einige Punkte festhalten.

Die Strategie der Haushaltsentlastung über Beteiligungen ist kein Charakteristikum unserer Zeit. Im Gegenteil wurde die Grenze zwischen Kernverwaltung, kommunaler Wirtschaft und Privatwirtschaft im vergangenen Jahrhundert immer wieder neu abgesteckt. Dahinter verbargen sich zum Teil wandelnde Leitbilder, stets aber auch wirtschaftliche Erwägungen. Die aktuellen Vorgänge der Rekommunalisierung bestätigen diese These. Auch hier treffen, wie in der Vergangenheit, sachliche Aspekte mit einem gewandelten Leitbild und haushalterischen Erwägungen zusammen. Es zeigt sich, dass sich die Rolle kommunaler Unternehmen in der Haushaltspolitik

9 Abweichend Bogumil 2010, S. 84 zur Rekommunalisierung in der Abfallentsorgung in Nordrhein-Westfalen. Gleichwohl muss bei solchen Studien berücksichtigt werden, welche Position in einer Stadtverwaltung um eine Einschätzung gebeten wurde. Es ist denkbar, dass der an Einnahmen interessierte Kämmerer Privatisierungen positiver einschätzt als die Politik, welche anderen Rationalitäten folgt.

10 Auslaufende Konzessionsverträge müssen öffentlich bekannt gemacht werden. In den ersten fünf Monaten des Jahres 2009 wurden 900 Bekanntmachungsanzeigen im Bundesanzeiger gezählt. Vgl. Verband Kommunaler Unternehmen 2010, S. 4. 
der Kommunen historisch wandelte. Stets hatten sie jedoch neben der eigentlichen Sachaufgabe eine fiskalische Funktion.

Unbestritten nehmen die kommunalen Unternehmen in der Konsolidierung der Kernhaushalte einen großen Stellenwert ein. Dieser lässt sich jedoch nicht klar in Zahlen ausdrücken (vgl. Truger 2008). Die rein finanzstatistischen Zahlungsbewegungen spiegeln die Bedeutung der Unternehmen nicht vollständig wider und können unterschiedlich interpretiert werden. Dabei lässt sich aus den Zahlen ablesen, dass Verkäufe eher auf Ebene von Bund und Ländern relevant waren, während die Kommunen formell privatisierten und damit organisatorische Optionen im Konzern schufen. Inwieweit Verkaufserlöse das kommunale Defizit spürbar reduzierten und zentraler Grund der Veräußerung waren, bleibt diskutabel. Tatsächlich sind die Zusammenhänge zwischen Kernverwaltung, Haushaltskrise und Unternehmen sehr viel komplexer. Sie drücken sich aus in mannigfaltigen Varianten, Aufgaben und Zahlungsströme im Konzern zu verteilen und damit in der Summe eine höhere Wirtschaftlichkeit zu erreichen. Das Ziel der Haushaltsentlastung gilt als wichtigstes Argument zurückliegender Auslagerungen. Inwieweit dies erreicht wurde, die Unternehmen tatsächlich wirtschaftlicher arbeiten, lässt sich hingegen nur vermuten, da der Vergleich mit dem Status quo ante naturgemäß nicht mehr möglich ist. Der Wandel hin zu ökonomischen Rationalitäten ist bei allen unintendierten Folgen ein Indiz wirtschaftlichen Erfolgs. Die Steigerung der Wirtschaftlichkeit öffentlicher Verwaltung als ein wesentliches Ziel der Auslagerungen ist darüber hinaus kein illegitimes Ziel, sondern sowohl notwendig angesichts beständig neuer Aufgaben als auch Verfassungsauftrag (vgl. Grundgesetz Artikel 114 Absatz 2). ${ }^{11}$

In diesem Sinne ist die Integration der Unternehmen in die Haushaltskonsolidierung nicht überraschend. Sie ist ebenso kein neues Phänomen, sondern ging stets mit kommunaler Wirtschaft einher. Deren Rolle hat sich daher nicht grundsätzlich geändert. Lediglich die Schwerpunkte zwischen Sach- und Finanzauftrag mögen sich vor dem Hintergrund der Haushaltskrise verschoben haben. Die Krise der Kommunen ist jedoch primär eine Krise der Kernhaushalte und Kernverwaltungen, deren Einnahmepotentiale begrenzt sind und für welche die Kommunalaufsicht spürbar war. Paradox kann im Vergleich das parallele Wachstum kommunaler Wirtschaft erscheinen. Doch die Strategie, Aufgaben aus der Kernverwaltung auszulagern, war angesichts vermeintlich besserer Ressourcenausstattung der Unternehmen rational.

Inwieweit Auslagerungen wirtschaftlich erfolgreich verliefen, ist in erster Linie eine Frage der Steuerung durch die Kernverwaltung, welche über viele Jahre als zu schwach galt. ${ }^{12}$ Wo sie vorhanden ist, ist sie häufig auf fiskalische Kennzahlen fokussiert. Hierin liegt die Gefahr, den Effizienzdruck auf die Unternehmen aus politischen Gründen über das betriebswirtschaftliche Maß hinaus zu erhöhen. Denn in Stadtrat und Kernverwaltung gelten die Unternehmen als politisch bequeme Option des Haushaltsausgleichs. Die Unternehmen werden, dies auch ein Indiz für ihre Bedeutung in der Haushaltskrise, tendenziell finanziell über- und inhaltlich untersteuert. Selbstverständlich darf sich der legitimierende öffentliche Zweck kommunaler Unternehmen nicht darin begrenzen, Instrument der Haushaltskonsolidierung zu sein.

11 Spezifiziert wird diese allgemeine Vorgabe in $\S 6$ des Haushaltsgrundsätzegesetzes in der Fassung vom 27.5.2010 (BGB1. I, S. 671).

12 Vergleichbar offen bleibt die Frage nach volkswirtschaftlichen Effekten. Vgl. Truger 2008. 
Mit der Komplexität der Kommunalverwaltung steigen Intransparenz und Risiken aus den Beteiligungen. Die Entfremdung zwischen Kernverwaltung, Politik und Unternehmen ist durchaus beidseitig, da der Gedanke einer wirklichen Public Governance über alle Einheiten des Konzerns noch nicht ausreichend entwickelt ist.

\section{Abstract}

René Geißler; Local public enterprises in cutback management

Budget Crisis; Cutback Management; local public enterprises; New Public Management; Privatization; Remunicipalisation;

Against the background of local budget crisis local enterprises as essential part of local self governance attracted more and more notice. This article addresses interrelations of local enterprises and budget consolidation. Recently this discussion was often simplified and ideological imprinted by the term privatization. This debate does not express local empiricism, which chose flexible options to integrate local enterprises in budget consolidation. Nevertheless the numerable effect in finance statistics is rather low. By far more important are disburdens of local budgets by a reorganization of functions within the local corporate group. Unfortunately these disburding effects are hardly to quantify and guided as by rising challenges in steering local enterprises as by fragmented budgets. Remunipicalisation is a rather recent trend in Germany. It is predominantly caused by the running out of a large share of concession contracts in utilities. Nonetheless it also follows fiscal arguments. But the fiscal relevance of local enterprises is not a present phenomenon. It was always existing.

\section{Literaturverzeichnis}

Ambrosius, Gerhard (1994), Die historisch wirksamen Kräfte bei der Entwicklung öffentlicher Unternehmen: Deutschland im internationalen Vergleich, in: Standortbestimmung öffentlicher Unternehmen in der Sozialen Marktwirtschaft, Hrsg. Eichhorn, Peter und Werner Wilhelm Engelhardt, S. 199-216, Baden-Baden: Nomos Verlag.

Ambrosius, Gerhard (2003), Das Verhältnis von Staat und Wirtschaft in historischer Perspektive: vornehmlich im Hinblick auf die kommunale Ebene, in: Die Ökonomisierung des öffentlichen Sektors: Instrumente und Trends, Hrsg. Harms, Jens und Christoph Reichard, S. 29-46, Baden-Baden: Nomos Verlag.

Bardt, Hubertus und Winfried Fuest (2007), Die wirtschaftliche Betätigung der Kommunen. Institut für Wirtschaft, Köln: IfW.

Bertelsmann Stiftung (2008), Kommunaler Schuldenreport, Gütersloh: Bertelsmann Stiftung.

Bieling, Hans-Jürgen (2008), Liberalisierung und Privatisierung in Deutschland. Versuch einer Zwischenbilanz, WSI Mitteilungen Nr. 10, Düsseldorf: WSI.

Bogumil, Jörg und Lars Holtkamp (2002), Liberalisierung und Privatisierung kommunaler Aufgaben. Auswirkungen auf das kommunale Entscheidungssystem, in: Liberalisierung und Privatisierung öffentlicher Aufgabenerfüllung. Soziale und umweltpolitische Perspektiven im Zeichen des Wettbewerbs, Hrsg. Libbe, Jens; Tomerius, Stephan und Hendrik Tropp, S. 71-91, Berlin: DIfU.

Bogumil, Jörg und Lars Holtkamp (2006), Kommunalpolitik und Kommunalverwaltung. Eine policyorientierte Einführung, Wiesbaden: VS Verlag.

Bogumil, Jörg; Grohs, Stefan; Kuhlmann, Sabine und Anna K. Ohm (2007), Zehn Jahre neues Steuerungsmodell. Bilanz kommunaler Verwaltungsmodernisierung, Berlin: Edition Sigma. 
Bogumil, Jörg; Pielow, Johann-Christian; Ebbinghaus, Jens; Gerber, Sascha und Maren Kohrsmeyer (2010), Die Gestaltung kommunaler Daseinsvorsorge im Europäischen Binnenmarkt. Empirische Untersuchung zu den Auswirkungen des europäischen Beihilfe- und Vergaberechts insbesondere im Abwasser- und Krankenhaussektor sowie in der Abfallentsorgung. Düsseldorf: Minister für Bundesangelegenheiten, Europa und Medien des Landes Nordrhein-Westfalen.

Bremeier, Wolfgang (1998), Kommunales Beteiligungsmanagement. Konzeptionen und Erfahrungen. In: Organisationswandel öffentlicher Aufgabenwahrnehmung, Hrsg. Dietrich Budäus, S. 287-317, Baden-Baden: Nomos Verlag.

Bremeier, Wolfgang (2000), Verwaltung 2000. Kommunale Unternehmen im Wettbewerb, in: Die erfolgreiche Steuerung öffentlicher Verwaltungen, Hrsg. Achim Töpfer, S. 325-338, Wiesbaden: Thomas Gabler Verlag.

Budäus, Dietrich (2000), Privatisierung öffentlich wahrgenommener Aufgaben, in: Privatisierung von Staatsaufgaben: Kriterien, Grenzen, Folgen, Hrsg. Christoph Gusy, S. 12-36, Baden-Baden: Nomos Verlag.

Budäus, Dietrich und Dennis Hilgers (2009), Reform des öffentlichen Haushalts- und Rechnungswesens in Deutschland. Konzepte, Umsetzungsstand und Entwicklungsperspektiven, in: Zeitschrift für Planung und Unternehmenssteuerung. S. 377-396.

Candeias, Mario; Rilling, Rainer und Katharina Weise (2008), Krise der Privatisierung. Rückkehr des Öffentlichen, in: WSI Mitteilungen Nr. 10. S. 563-569.

Caspari, Ingo (2009), Positionen zu Privatisierungen. Wissenschaftliche und politische Einstellungen und ihre Bedeutung für das kommunale Handeln, Frankfurt am Main: Peter Lang Verlag.

Deckwirth, Christina (2008), Die Europäische Union als Triebkraft der Privatisierung, WSI-Mitteilungen Nr. 10 , S. 534-547.

DiMaggio, Paul und Walter Powell (1983), The Iron Cage revisited: Institutional Isomorphism and collective Rationality in organizational Fields, in: American Sociological Review. No. 4. S. 147-160.

Edeling, Thomas (2003), Rollenverständnis des Managements im kommunalen Unternehmen, in: Die Ökonomisierung des öffentlichen Sektors: Instrumente und Trends, Hrsg. Harms, Jens und Christoph Reichard, S. 235-254, Baden-Baden: Nomos Verlag.

Edeling, Thomas (2004), Umstrittene Grenzen. Zur Standortbestimmung öffentlicher Unternehmen, in: Öffentliche Unternehmen zwischen Privatwirtschaft und öffentlicher Verwaltung. Eine empirische Studie im Feld kommunaler Versorgungsunternehmen, Hrsg. Edeling, Thomas; Stölting, Erhard und Dieter Wagner, S. 9-41, Wiesbaden: VS Verlag.

Eichhorn, Peter (1997), Ausgliederung als Instrument zur Flexibilisierung kommunaler Aufgabenerfüllung, in: Privatisierung kommunaler Aufgaben, Hrsg. Fettig, Wolfgang und Lothar Späth, S. 96-102, Baden-Baden: Nomos Verlag.

Engartner, Tim (2009), Kehrt der Staat zurück? Rekommunalisierungen in den Bereichen Entsorgung und Gebäudereinigung, in: ZögU Nr. 4, S. 339-335.

Geißler, Rene (2009), Kommunalrechtliche Steuerungsansätze der Haushaltskonsolidierung. KWI Gutachten, Potsdam: Universitätsverlag.

Geißler, René (2011 a), Innovationsmanagement in der kommunalen Haushaltskonsolidierung, in: Innovative Verwaltungen. Innovationsmanagement als Instrument von Verwaltungsreformen, Hrsg. Schauer, Reinbert; Thom, Norbert und Dennis Hilgers, S. 521-534, Linz: Trauner Verlag.

Geißler, René (2011 b), Kommunale Haushaltskonsolidierung. Einflussfaktoren lokaler Konsolidierungspolitik. Wiesbaden: VS Verlag.

Gern, Alfons (2004), Deutsches Kommunalrecht. 3. Auflage. Baden-Baden: Nomos Verlag.

Gottschalk, Wolf (1994), Kommunale Unternehmen als Instrumente der Selbstverwaltung, in: Standortbestimmung öffentlicher Unternehmen in der Sozialen Marktwirtschaft, Hrsg. Eichhorn, Peter und Werner Wilhelm Engelhardt, S. 217-226, Baden-Baden: Nomos Verlag.

Gottschalk, Wolf (2003), Auswirkungen von Liberalisierung und Privatisierung: Kommerzialisierung kommunaler Unternehmen, in: Die Ökonomisierung des öffentlichen Sektors. Instrumente und Trends, Hrsg. Harms, Jens und Christoph Reichard, S. 201-212, Baden-Baden: Nomos Verlag.

Greiling, Dorothea (2011), Ausgliederungen und Public Private Partnerships als Instrumente zur Erfüllung öffentlicher Aufgaben. ein österreichischer Erfahrungsbericht, in: ZögU Nr. 1, S. 52-80.

Gusy, Christoph (Hrsg.) (1998), Privatisierung von Staatsaufgaben: Kriterien, Grenzen, Folgen, Baden-Baden: Nomos Verlag.

Hammerschmid, Gerhard (2009, Public Corporate Governance. Modewelle oder tatsächlicher Bedarf? in: Zukunft der öffentlichen Wirtschaft. Beiträge zur öffentlichen Wirtschaft Heft 31, Hrsg. Bundesverband Öffentliche Dienstleistungen, S. 5-16, Berlin: Gesellschaft für öffentliche Wirtschaft.

Hardrath, Klaus (2003), Zur Sanierung öffentlicher Haushalte durch Privatisierung öffentlicher Aufgaben, in: Sächsische Verwaltungsblätter Nr. 3, S. 53-61.

Harms, Jens und Christoph Reichard (2003), Ökonomisierung des öffentlichen Sektors. Eine Einführung, in: Die Ökonomisierung des öffentlichen Sektors: Instrumente und Trends. Hrsg. dies., S. 13-18, Baden-Baden: Nomos Verlag. 
Henke, Hans Joachim; Hillebrand, Rainer und Silke Steltmann (2007), Müssen öffentliche Unternehmen anders gesteuert werden als private Unternehmen? in: Public Corporate Governance. Ein Kodex für öffentliche Unternehmen, Hrsg. Ruter, Rudolf X.; Sahr, Karin und Georg Waldersee, S. 111-116, Wiesbaden: Gabler Verlag.

Holtkamp, Lars (2007), Wer hat Schuld an der Verschuldung? Ursachen nationaler und kommunaler Haushaltsdefizite, polis Nr. 64. Hagen: Fernuniversität Hagen.

Holtkamp, Lars (2008), Das Scheitern des Neuen Steuerungsmodells, in: Der moderne Staat, Nr. 2, S. $423-446$.

Jann, Werner (2010), Neues Steuerungsmodell, in: Handbuch zur Verwaltungsreform, Hrsg. Blanke, Bernhard; Nullmeier, Frank; Reichard, Christoph und Göttrik Wewer, 4. Auflage, S. 98-108, Wiesbaden: VS Verlag.

Kilian, Werner; Richter, Peter und Jan Hendrik Trapp (2006), Einleitung, in: Ausgliederung und Privatisierung in Kommunen, (Hrsg.) Kilian, Werner; Richter, Peter und Jan Hendrik, S. 9-24, Berlin: Edition Sigma.

Kurth, Peter (1999), Ausgliederung und Privatisierung als Mittel der Haushaltskonsolidierung, in: Kommunale Wirtschaft im Wandel, Hrsg. Eichhorn, Peter; Schuppert, Gunnar F. und Christoph Reichard, S. 129-139, Berlin: Gesellschaft für öffentliche Wirtschaft.

Lenk, Thomas; Rottmann, Oliver und Romy Albrecht (2011 a), Renaissance der Kommunalwirtschaft, Rekommunalisierung öffentlicher Dienstleistungen, Leipzig: Institut für Öffentliche Finanzen und Public Management.

Lenk, Thomas; Rottmann, Oliver und Romy Albrecht (2011 b): Rekommunalisierung der Energieversorgung, in: Public Governance. S. 6-11.

Le Nestour, Chantal (2001), Elektrizitätswirtschaft, in: Die öffentliche Wirtschaft in Deutschland Bestandsaufnahme zu Beginn des 21. Jahrhunderts, Hrsg. Gesellschaft für öffentliche Wirtschaft, S 28-31, Berlin: Bundesverband Öffentliche Dienstleistung.

Leonhardt, Willy (2000), Kommunale Strategien als Antwort auf die Liberalisierung der Märkte, in: Kommunale Wirtschaft im Wandel. Risiken und Chancen, Hrsg. Eichhorn. Peter; Reichard, Christoph und Gunnar Folke Schuppert, S. 61-85, Baden-Baden: Nomos Verlag.

Löwe, Jürgen (2001), Öffentliche Unternehmen in der Marktwirtschaft? Ein Beitrag zur Neubestimmung des Verhältnisses von Wirtschaft und Politik, in: ZögU Nr. 4, S. 413-431.

Mezger, Erika und Karsten Schneider (2006), Die Grenzen privater Beteiligung an öffentlichen Aufgaben. In: Public Management: Grundlagen, Wirkungen, Kritik, Hrsg. Jann, Werner; Röber, Manfred und Helmut Wollmann, S. 49-59, Berlin: Edition Sigma.

Monopolkommission (2009), Strom und Gas 2009. Energiemärkte im Spannungsfeld von Politik und Wettbewerb, Sondergutachten 54, Bonn: Monopolkommission.

Mühlenkamp, Holger (2001), Rechtsform, Trägerschaft und Effizienz öffentlicher Unternehmen. Eine Untersuchung der Auswirkungen formeller Privatisierungsmaßnahmen am Beispiel öffentlicher Theater mit Hilfe von PanelDaten, in: ZögU 2, S. 152-169.

Naßmacher, Hiltrud und Karl-Heinz Naßmacher (1999), Kommunalpolitik in Deutschland, Opladen: Leske und Budrich.

Oettle, Karl (1994), Gemeinsame Spartenfinanzierung in Querverbundunternehmen. Zweck-mäßige Alimentierungen oder marktwidrige Subventionierungen? in: Standortbestimmung öffentlicher Unternehmen in der Sozialen Marktwirtschaft, Hrsg. Eichhorn, Peter und Werner Wilhelm Engelhardt, S. 297-309, Baden-Baden: Nomos Verlag.

Papenfuß, Ulf (2010), Unmittelbare Beteiligungen der öffentlichen Hand in der Kontroverse um Daseinsvorsorge. Eine empirische Analyse von allen im Internet verfügbaren Beteiligungsberichten zu Anzahl und Rechtsformen, in: ZögU Nr. 2, S. 97-121.

Püttner, Günter (1994), Privilegierung oder Diskriminierung öffentlicher Unternehmen im Europarecht, in: Standortbestimmung öffentlicher Unternehmen in der Sozialen Marktwirtschaft, Hrsg. Eichhorn, Peter und Werner Wilhelm Engelhardt, S. 339-348, Baden-Baden: Nomos Verlag.

Reichard, Christoph (2000), Kommunale Wirtschaft unter Anpassungsdruck, in: Kommunale Wirtschaft im Wandel. Chancen und Risiken, Hrsg. Eichhorn, Peter; Reichard, Christoph und Gunnar Folke Schuppert, S. 15-24, BadenBaden: Nomos Verlag.

Reichard, Christoph (2004), Die Stadt als Konzern. Corporatization als Fortführung des NSM? in: Perspektiven kommunaler Verwaltungsmodernisierung. Praxiskonsequenzen aus dem Neuen Steuerungsmodell, Hrsg. Bogumil, Jörg; Holtkamp, Lars; Banner, Gerhard und Leo Kißler, S. 55-64, Berlin: Edition Sigma.

Reidenbach, Michael (2009), Investitionsstau und Investitionsbedarf. In: WSI Mitteilungen Nr. 5, S. 251-259.

Richter, Walter (1995), Effiziente Nutzung von kommunalem Vermögen als Haushaltskonsolidierungsansatz, in: Sparstrategien, Hrsg. Birgit Frischmuth, S. 72-78, Berlin: DIfU.

Richter, Peter; Kilian, Werner und Hendrik Trapp (2006), Verselbständigung kommunaler Aufgabenerbringung und die Folgen, in: Ausgliederung und Privatisierung in Kommunen, Hrsg. Richter, Peter; Kilian, Werner und Hendrik Trapp, S. 111-130, Berlin: Edition Sigma.

Richter, Peter (2007), Die Bedeutung der kommunalen Wirtschaft. Eine vergleichende Ost-West-Analyse, Berlin: Edition Sigma.

Richter, Peter und Thomas Edeling (2011), Zwischen Entlastung und dem Traum von Wohlstand. Privatisierung kommunaler Unternehmen in strukturschwachen Regionen Brandenburgs, Potsdam: Universitätsverlag. 
Röber, Manfred (2009), Daseinsvorsorge zwischen Privatisierung und Kommunalisierung. Anmerkungen aus der Perspektive des Public Management, in: Zukunft der öffentlichen Wirtschaft, Hrsg. Bundesverband öffentliche Dienstleistungen, S. 74-101, Baden-Baden: Nomos Verlag.

Rollenfitsch, Michael (2007), Entwicklung und Tendenzen privatwirtschaftlicher Betätigung der Gemeinden, in: Handbuch kommunale Unternehmen, Hrsg. Uechtritz, Michael und Werner Hoppe, S. 3-27, Köln: Verlag Dr. Otto Schmidt.

Sack, Detlef (2006), Liberalisierung und Privatisierungen in den Kommunen. Steuerungsanforderungen und Folgen für Entscheidungsprozesse, in: Deutsche Zeitschrift für Kommunal-wissenschaften Nr. 2, S. 25-38.

Schmitt, Carina (2011), Der Staat als Unternehmer bei der Bereitstellung öffentlicher Dienstleistungen. Konvergenz im internationalen Vergleich? in: Der moderne Staat Nr. 1, S. 209-224

Schmitt, Carina und Herbert Obinger (2010), Verfassungsschranken und die Privatisierung öffentlicher Dienstleistungen im internationalen Vergleich, in: Politische Vierteljahresschrift Nr. 4, S. 643-664.

Scholle, Manfred (2010), Der Trend zur Rekommunalisierung. Chance oder Risiko? in: Nachhaltige Kommunalpolitik. Ein Anforderungsprofil, Hrsg. Gernert, Wolfgang; Konegen, Nobert und Reinhard Meyers, S. 95-108, Münster: Waxmann Verlag.

Schwarting, Gunnar (2001), Kommunale Wirtschaft. Vor großen Herausforderungen, in: ZögU Nr. 3, S. 287-307.

Schwarting, Gunnar (2006), Haushaltskonsolidierung in Kommunen. Ein Leitfaden für Rat und Kommune, 2. Auflage, Berlin: Erich Schmidt Verlag.

Schubert, Annegret (2010), Die Privatisierung des E-Government, Stuttgart: Verlag Boorberg.

Schwintowski, Hans-Peter (2003), Gemeinwohl, öffentliche Daseinsvorsorge und Funktionen öffentlicher Unternehmen im europäischen Binnenmarkt, In: ZögU Nr. 3, S. 283-309.

Statistisches Bundesamt (2011), Datenreport 2011, Wiesbaden: Statistisches Bundesamt.

Strünck, Christoph und Rolf Heinze (2005), Public Private Partnership, in: Handbuch der Verwaltungsreform, Hrsg. Blanke, Bernhard; von Bandemer, Stephan; Nullmeier, Frank und Göttrik Wewer, 3. Auflage, S. 120-128, Wiesbaden: VS Verlag.

Theuvsen, Ludwig und Ulrike Zschache (2011), Die Privatisierung kommunaler Unternehmen im Spiegel massenmedialer Diskurse, in: ZögU Nr. 1, S. 3-24.

Trappe, Jan Hendrik und Sebastian Bolay (2003), Privatisierung in Kommunen. eine Auswertung kommunaler Beteiligungsberichte, Berlin: DIfU.

Truger, Achim (2008), Privatisierung und öffentliche Finanzen, in: WSI Mitteilungen Nr. 10, S. 548-555.

van Loesch, Achim (1997), Die öffentlichen Unternehmen Deutschlands, in: Öffentliche Verwaltung in Deutschland, Hrsg. König, Klaus und Heinrich Siedentopf, S. 285-300, 2. Auflage, Baden-Baden: Nomos Verlag.

Verband kommunaler Unternehmen (2010), Konzessionsverträge. Handlungsoptionen für Kommunen und Stadtwerke. Berlin: Verband kommunaler Unternehmen.

Wegrich, Kai (2010), Post-New Public Management, in: Handbuch zur Verwaltungsreform, Hrsg. Blanke, Bernhard; Nullmeier, Frank; Reichard, Christoph und Göttrik Wewer, 4. Auflage, Wiesbaden 2010, S. 90-97.

Wissenschaftlicher Beirat der Gesellschaft für Öffentliche Wirtschaft und Gemeinwirtschaft (1979), Privatisierung öffentlicher Unternehmen. Kein Mittel zum Abbau von Haushaltsdefiziten. Stellungnahme zum Gutachten des Wissenschaftlichen Beirats beim Bundesministerium der Finanzen zur Lage und Entwicklung der Staatsfinanzen in der Bundesrepublik Deutschland. Berlin: Gesellschaft für öffentliche Wirtschaft. 